\title{
Advances in optical engineering for future telescopes
}

\author{
Daewook Kim ${ }^{1,2,3 *}$, Heejoo Choi $^{1,3}$, Trenton Brendel ${ }^{1}$, Henry Quach ${ }^{1}$, \\ Marcos Esparza ${ }^{1}$, Hyukmo Kang ${ }^{1}$, Yi-Ting Feng ${ }^{1}$, Jaren N. Ashcraft ${ }^{1}$, \\ Xiaolong $\mathrm{Ke}^{4}$, Tianyi Wang ${ }^{5}$ and Ewan S. Douglas ${ }^{2}$
}

Significant optical engineering advances at the University of Arizona are being made for design, fabrication, and construction of next generation astronomical telescopes. This summary review paper focuses on the technological advances in three key areas. First is the optical fabrication technique used for constructing next-generation telescope mirrors. Advances in ground-based telescope control and instrumentation comprise the second area of development. This includes active alignment of the laser truss-based Large Binocular Telescope (LBT) prime focus camera, the new MOBIUS modular cross-dispersion spectroscopy unit used at the prime focal plane of the LBT, and topological pupil segment optimization. Lastly, future space telescope concepts and enabling technologies are discussed. Among these, the Nautilus space observatory requires challenging alignment of segmented multi-order diffractive elements. The OASIS terahertz space telescope presents unique challenges for characterizing the inflatable primary mirror, and the Hyperion space telescope pushes the limits of high spectral resolution, far-UV spectroscopy. The Coronagraphic Debris and Exoplanet Exploring Pioneer (CDEEP) is a Small Satellite (SmallSat) mission concept for high-contrast imaging of circumstellar disks and exoplanets using vector vortex coronagraph. These advances in optical engineering technologies will help mankind to probe, explore, and understand the scientific beauty of our universe.

Keywords: computer controlled optical surfacing; CCOS multiplexing; dwell time optimization; optical metrology; telescope alignment; large binocular telescope; MOBIUS; pupil segmentation; OASIS; nautilus; hyperion; CDEEP; vector vortex coronagraph

Kim D, Choi H, Brendel T, Quach H, Esparza M et al. Advances in optical engineering for future telescopes. Opto-Electron Adv 4, 210040 (2021).

\section{Introduction}

Astronomical advances are largely coupled with technological improvements. From the invention of the first optical telescope used by Galileo in 1609 and through the foreseeable future, astronomy and optical engineering are forever linked. In this paper, several advances are summarized that will enable future telescopes to probe and expand our scientific understanding of the universe.

To begin, Section Deterministic computer controlled optical surfacing technologies covers optical fabrication

${ }^{1}$ Wyant College of Optical Sciences, University of Arizona, 1630 E. University Blvd., Tucson, AZ 85721, USA; ${ }^{2}$ Department of Astronomy and Steward Observatory, University of Arizona, 933 N. Cherry Ave., Tucson, AZ 85721, USA; ${ }^{2}$ Large Binocular Telescope Observatory, University of Arizona, 933 N. Cherry Ave., Tucson, AZ 85721, USA; ${ }^{4}$ School of Mechanical and Automotive Engineering, Xiamen University of Technology,

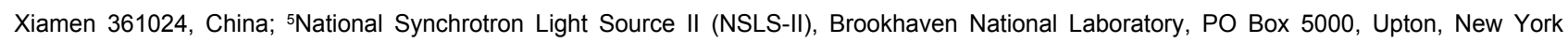
11973, USA.

'Correspondence: D Kim, E-mail: dkim@optics.arizona.edu

Received: 29 March 2021; Accepted: 20 May 2021; Published: 20 June 2021

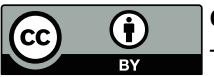

Open Access This article is licensed under a Creative Commons Attribution 4.0 International License.

To view a copy of this license, visit http://creativecommons.org/licenses/by/4.0/.

(C) The Author(s) 2021. Published by Institute of Optics and Electronics, Chinese Academy of Sciences. 
and manufacturing process optimization technologies. Fabrication of large optics is an extremely time-consuming process due to the large size and high-accuracy requirements ${ }^{1-4}$. For example, polishing each segment of the Giant Magellan Telescope (GMT) to the specified accuracy consumed about one year. Modern precision optical surfaces are manufactured with Computer Controlled Optical Surfacing (CCOS) processes $^{5-7}$, such as small-tool polishing ${ }^{5,7}$, bonnet polishing ${ }^{8}$, magnetorheological finishing, ion beam figuring ${ }^{9,10}$, etc. With these systems, there are two main categories of methods for improving polishing efficiency: increasing the material removal rate and minimizing the total dwell time. In this section, both categories are addressed. The mathematical theory of multiplexed two CCOS runs for simultaneous use of two polishing tools has been developed ${ }^{6}$. Dwell time optimization is demonstrated via Robust Iterative Fourier Transform-based dwell time Algorithm (RIFTA) ${ }^{9}$. These emerging technologies provide opportunity to increase speed and reduce cost of large optics manufacturing.

Section Very large telescope control system, instrument, and segmentation summarizes optical engineering technology developments for very large telescopes. As a first example, the Large Binocular Telescope (LBT) team is developing a laser-truss based alignment system for maintaining telescope collimation and pointing ${ }^{11,12}$. The laser truss metrology system, able to measure the relative position of optics in six degrees of freedom, is the first of its kind to be implemented on large, ground-based telescopes. A second innovation for the LBT system is an instrument package referred to as MOBIUS (Mask-Oriented Breadboard Implementation for Unscrambling Spectra). The MOBIUS system adds cross-dispersion capability to the existing LUCI (LBT Utility Camera in the Infrared) near infrared (NIR) imagers and spectrographs ${ }^{13}$. By inserting the MOBIUS module, we extend simultaneous coverage down to $0.32 \mu \mathrm{m}$ by binocular observations with one MODS (The Multi-Object Double Spectrographs) and one LUCI. The final example discussed in Section Very large telescope control system, instrument, and segmentation is segmentation of primary mirror apertures. A pinwheel aperture segmentation is a promising approach which obtains a point spread function (PSF) with enhanced axial symmetry. This is modeled using Fraunhofer diffraction theory with the Python-based POPPY simulation tool ${ }^{14}$.

Section Future space telescope concepts and enabling technologies introduces some next generation space telescope concepts. The multi-order diffractive engineered (MODE) lens is a novel optical element that is lightweight, achromatic across a large spectrum and has more relaxed tolerances than mirror segments of a similar size $^{15-19}$. The Nautilus Space Observatory concept provides a new solution utilizing the transmissive MODE lens segments. Our group has developed both a mechanism to align MODE lens segments ${ }^{20}$ and an inprocess metrology system to monitor the lens alignment while the segments are being bonded together ${ }^{21}$. The Orbiting Astronomical Satellite for Investigating Stellar Systems (OASIS) is a proposed terahertz space observatory that will explore the role of water in the evolution of planetary systems and features a 20-meter diameter primary mirror, which is obtained by using inflatable membrane technology ${ }^{19,22-24}$. By pressurizing one transparent and one metallized membrane sealed around a rigid tensioning ring, a lenticular surface with immense light-collecting power can be obtained. We present a metrology technique for measuring the surface figure of such novel inflatable mirrors ${ }^{24}$. At the other end of the optical spectrum, the next generation UV space telescope concept, Hyperion, is designed to examine the origin of the star formation ${ }^{25,26}$. At the atomic-to-molecular interstellar boundary layer, the fuel for star formation (SF) exists in a state of $\mathrm{H} 2$. Both the reflective telescope and cross-dispersion instrumentation design using freeform optics are presented. The Coronagraphic Debris and Exoplanet Exploring Pioneer (CDEEP) is a SmallSat mission concept for high-contrast imaging of circumstellar disks and exoplanets. CDEEP is equipped with a vector vortex coronagraph (VVC) to achieve the contrast necessary to resolve debris disks in scattered light at visible wavelengths $\left(<10^{-8}\right.$ with post processing). This instrument will enable the classification of transport-dominated debris disks by measuring the albedo, composition, and morphology with extreme sensitivity. The design of the CDEEP mission and associated vacuum compatible high-contrast imaging testbed are documented in Maier et $\mathrm{al}^{27}$.

The authors acknowledge that this invited summary paper is significantly based on and directly overlaps with many parts of a previous conference publication ${ }^{28}$. The goal of this extended review article is to provide comprehensive details and substantial updates with additional/ reorganized materials presenting the latest optical engineering technology developments conducted at the University of Arizona. 


\section{Deterministic computer controlled optical surfacing technologies}

Fabricating the large, complicated optical surfaces required by future telescopes is continuously being improved with innovative techniques to reduce cost and time. In this section, two approaches are discussed. Simultaneous polishing with multiple tools of different sizes provides a holistic approach to polishing because tool size is related to the spatial frequency of the surface errors that can be corrected. The other method, dwell time optimization, improves the efficiency of the removal by spending more effort on the high surface and minimizing time spent in regions with low error. Both techniques help make future telescopes a reality.

\section{Dual-tool multiplexed polishing model for computer controlled optical surfacing}

Many future telescope system designs utilize non-trivial optical surfaces and components such as freeform lens or mirrors in order to control the aberrations especially for wide field of view systems. Those precision optics manufacturing efficiency can be significantly improved if multiple fabrication tools are adopted simultaneously in a single polishing process. For instance, the Large Polishing Machine (LPM) in Fig. 1 consists of two tools with different sizes. A $1.2 \mathrm{~m}$ diameter stressed lap (labelled $\mathrm{Tool}_{1}$ ) and a $0.3 \mathrm{~m}$ diameter non-Newtonian lap (labelled $\mathrm{Tool}_{2}$ ) have been used in the LPM to ensure adequate removal for the Large Synoptic Survey Telescope (LSST) and GMT. They can be controlled simultaneously and independently during a single CCOS run.

In order to accelerate the polishing process speed, a dual-tool multiplexing polishing model enabling simultaneous running of the two polishing heads was de-

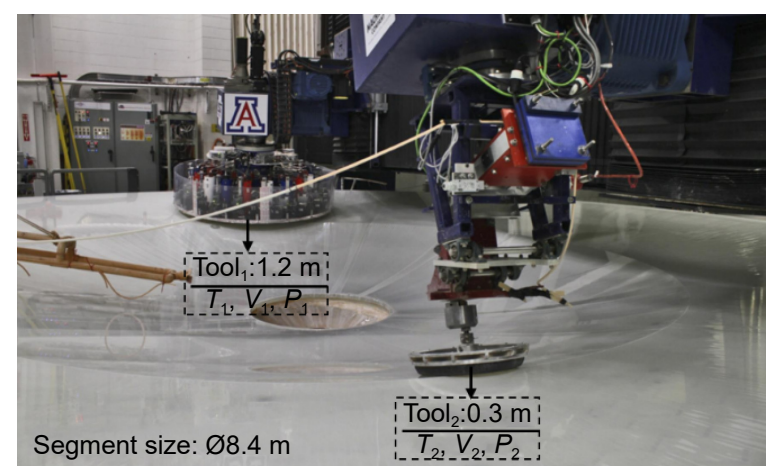

Fig. 1 Large polishing machine (LPM) with dual tools at the University of Arizona. Figure reproduced with permission from ref. ${ }^{6}$, Optical Society of America. veloped ${ }^{6}$. The convolutional material removal model of an individual tool is defined as

$$
Z(x, y)=R(u, v) * *[T(x, y) \times V(x, y) \times P(x, y)],
$$

where "**" represents the convolution operator; $Z(x, y)$ is the removed material distribution, which is equal to the convolution between the basic Tool Influence Function (TIF) $R(u, v)$ and the product of the dwell time $T(x, y)$, the velocity $V(x, y)$, and the contact pressures $P(x, y)$. Equation (1) is still valid in the dual-tool multiplexing model. Moreover, since the material removed at a certain dwell point in the single-tool model is required to be identical to that in the dual-tool model, the following boundary condition $\mathrm{BC}(\mathrm{BC}-1)$ should be fulfilled,

$$
\text { BC-1 : } Z_{1}^{s}(x, y) \equiv Z_{1}^{\mathrm{m}}(x, y) \quad \text { and } \quad Z_{2}^{s}(x, y) \equiv Z_{2}^{\mathrm{m}}(x, y) \text {, }
$$

where $Z_{1}^{\mathrm{s}}(x, y)$ and $Z_{2}^{\mathrm{s}}(x, y)$ represents the material removed by $R_{1}$ (the basic TIF of Tool ${ }_{1}$ ) and $R_{2}$ (the basic TIF of $\left.\mathrm{Tool}_{2}\right)$ at $(x, y)$ in the single-tool model, respectively; $Z_{1}^{\mathrm{m}}(x, y)$ and $Z_{2}^{\mathrm{m}}(x, y)$ represents the material removed by $R_{1}$ and $R_{2}$ at $(x, y)$ in the dual-tool model, respectively. Since $R_{1}$ and $R_{2}$ remain invariant, Eq. (2) can be further transformed into

$$
\left\{\begin{array}{l}
T_{1}^{\mathrm{s}} \times V_{1}^{\mathrm{s}} \times P_{1}^{\mathrm{s}}=T_{1}^{\mathrm{m}} \times V_{1}^{\mathrm{m}} \times P_{1}^{\mathrm{m}} \\
T_{2}^{\mathrm{s}} \times V_{2}^{\mathrm{s}} \times P_{2}^{\mathrm{s}}=T_{2}^{\mathrm{m}} \times V_{2}^{\mathrm{m}} \times P_{2}^{\mathrm{m}},
\end{array},\right.
$$

where $T_{1}^{\mathrm{s}}, V_{1}^{\mathrm{s}}$ and $P_{1}^{\mathrm{s}}$ are the dwell time, the velocity, and the contact pressure of $\mathrm{Tool}_{1}$ in the single-tool model, respectively; and $T_{2}^{\mathrm{s}}, V_{2}^{\mathrm{s}}$ and $P_{2}^{\mathrm{s}}$ are the dwell time, the velocity, and the contact pressure of $\mathrm{Tool}_{2}$ in the single-tool model, respectively. Similarly, $T_{1}^{\mathrm{m}}, V_{1}^{\mathrm{m}}, P_{1}^{\mathrm{m}}, T_{2}^{\mathrm{m}}, V_{2}^{\mathrm{m}}$ and $P_{2}^{\mathrm{m}}$ are the corresponding parameters for $\mathrm{Tool}_{1}$ and $\mathrm{Tool}_{2}$ in the dual-tool model.

Since LPM works in workpiece rotation mode (see Fig. 1), the simultaneous run of the two tools requires the dwell time for each individual tool to be synchronized. This is achieved by setting one tool (e.g. Tool ${ }_{1}$ ) as the primary tool so that its dwell time and velocities remain invariant, i.e.

$$
\left\{\begin{array}{l}
T_{1}^{\mathrm{s}}(x, y)=T_{1}^{\mathrm{m}}(x, y) \\
V_{1}^{\mathrm{s}}(x, y)=V_{1}^{\mathrm{m}}(x, y)
\end{array} .\right.
$$

In addition, during simultaneous polishing, Tool $_{1}$ and $\mathrm{Tool}_{2}$ are placed at the opposite locations (i.e. $180^{\circ}$ ). Therefore, $T_{2}^{\mathrm{m}}$ can be calculated with the machine-specific $\mathrm{BC}(\mathrm{BC}-2)$ as

$$
\text { BC-2: } T_{2}^{\mathrm{m}}(x, y) \equiv \operatorname{rotate}\left(T_{1}^{\mathrm{m}}(x, y), 180^{\circ}\right) \text {. }
$$

It is worth mentioning that the separation angle between $\mathrm{Tool}_{1}$ and $\mathrm{Tool}_{2}$ can be arbitrary. The $180^{\circ}$ is 
just the most convenient angle to implement the dualtool model under LPM's specific gantry-type configuration. Based on Eqs. (4) and (5), $V_{2}^{\mathrm{m}}$ is determined as:

$$
V_{2}^{\mathrm{m}}(x, y)=V_{2}^{\mathrm{s}}(x, y) \frac{T_{2}^{\mathrm{s}}(x, y)}{T_{2}^{\mathrm{m}}(x, y)} .
$$

As the contact pressure is often set as constants, all of the parameters for the dual-tool model are now synchronized. However, $V_{2}^{\mathrm{m}}(x, y)$ obtained from Eq. (6) may exceed the maximum velocity allowed by the polishing machine. Therefore, the Velocity Adjustment Algorithm (VAA) shown by the algorithm in Fig. 2 is employed to constrain the velocities under the valid range. VAA adjusts the velocities of $\mathrm{Tool}_{2}$ and $\mathrm{Tool}_{1}$ in two loops, respectively. In the first loop, $V_{2}^{\mathrm{m}}(x, y)$ is clamped to the $V_{2}^{\max }$ if it is over the range, and $T_{2}^{\mathrm{m}}(x, y), T_{1}^{\mathrm{m}}(x, y)$ and $V_{1}^{\mathrm{m}}(x, y)$ are updated accordingly. Similarly, in the second loop, $V_{1}^{\mathrm{m}}(x, y)$ is clamped to the $V_{1}^{\max }$ if it is over the range, and $T_{1}^{\mathrm{m}}(x, y), T_{2}^{\mathrm{m}}(x, y)$ and $V_{2}^{\mathrm{m}}(x, y)$ are updated accordingly.

The performances of the single-tool sequential polishing and dual-tool simultaneous polishing are studied using a simulated surface error map in shown in Fig. 3, where Figs. 3(a) and 3(b) are the results of two sequential single-tool runs. The figure error is reduced from $1.72 \mu \mathrm{m}$ RMS to $184.9 \mathrm{~nm}$ RMS after processed by Tool $_{1}$ (see Fig. 3(a)), which is then further decreased to $3.4 \mathrm{~nm}$ RMS by Tool 2 shown in Fig. 3(b). The total dwell time of the sequential runs is $22.62+36.54=59.16 \mathrm{~h}$.

In the dual-tool multiplexed model, two tool-feed modes are tested, namely the in-out mode shown in Figs. 3(d) and 3(f) and the in-in mode shown in Figs. 3(c) and $3(\mathrm{e})$. The in-out mode is generally applicable to any dualtool polishing scenario, because $\mathrm{Tool}_{1}$ and $\mathrm{Tool}_{2}$ move in the same direction and the tool collision can be avoided.

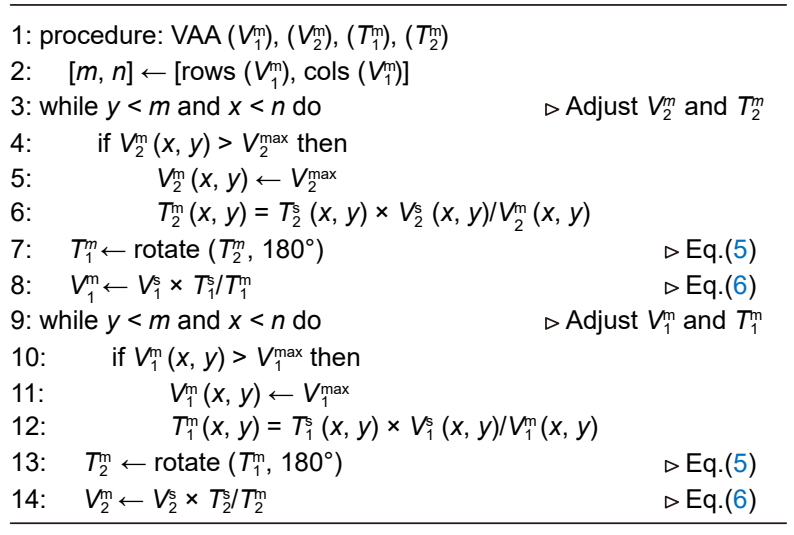

Fig. 2 | Flow of velocity adjustment algorithm. Figure reproduced with permission from ref. ${ }^{6}$, Optical Society of America.
The in-in mode has the potential problem of tool collision, however, it can also be applied for the GMT on-axis segment here, since this problem is automatically resolved because of the hole at the mirror's center. Tool ${ }_{1}$ is selected as the primary tool in the dual-tool polishing, so that the parameters of $\mathrm{Tool}_{2}$ (i.e. the dwell time and velocities) are adjusted and synchronized with those of Tool $_{1}$. The performances of the in-in and the in-out feed modes are studied with equal-angel and equal-arc-length path types, respectively. With the equal-angel paths, as shown in Figs. 3(c) and 3(d), the in-in feed mode achieves 29.26 $\mathrm{h}$, which is shorter than that $(37.08 \mathrm{~h})$ of the in-out feed mode. Compared with the single-tool sequential result, the polishing efficiency is improved by $50.54 \%$ with the in-in mode.

The two feed modes with the equal-arc-length paths are further demonstrated in Figs. 3(e) and 3(f). The total dwell time of the in-in feed mode shown in Fig. 3(e) is $29.26 \mathrm{~h}$, which achieves a similar residual error as those in Figs. 3(c) and 3(d). It is worth mentioning that, however, the in-out feed mode cannot be applied with the equal-arc-length path as shown in Fig. 3(f), since the two tools have different redial positions at any instant so that the arc lengths cannot be equal.

In a word, the multiplexed dual-tool deterministic polishing model can enable the efficient polishing of large optics. Also, the dual-tool multiplexing model can be further extended to an $\mathrm{N}$-tool model multiplexing $\mathrm{N}$ tools, in which case the polishing efficiency can be further improved.

\section{Robust iterative Fourier transform-based dwell time algorithm for CCOS}

One of the most essential numerical optimization problems of a CCOS process is to optimize the dwell time which controls how much time a tool dwells at a certain position on the surface of a workpiece. The dwell time optimization is guided by the convolutional polishing model $^{29}$,

$$
z(x, y)=b(x, y) * t(x, y),
$$

where " $*$ " represents the convolution operation, $b(x, y)$ is the Tool Influence Function (TIF), $z(x, y)$ is the removed material, and $t(x, y)$ is the dwell time. $z(x, y)$ and $b(x, y)$ are known, $t(x, y)$ is thus calculated via deconvolution, which is an ill-posed operation that may not result in a reliable and unique solution of $t(x, y)$. Equation 


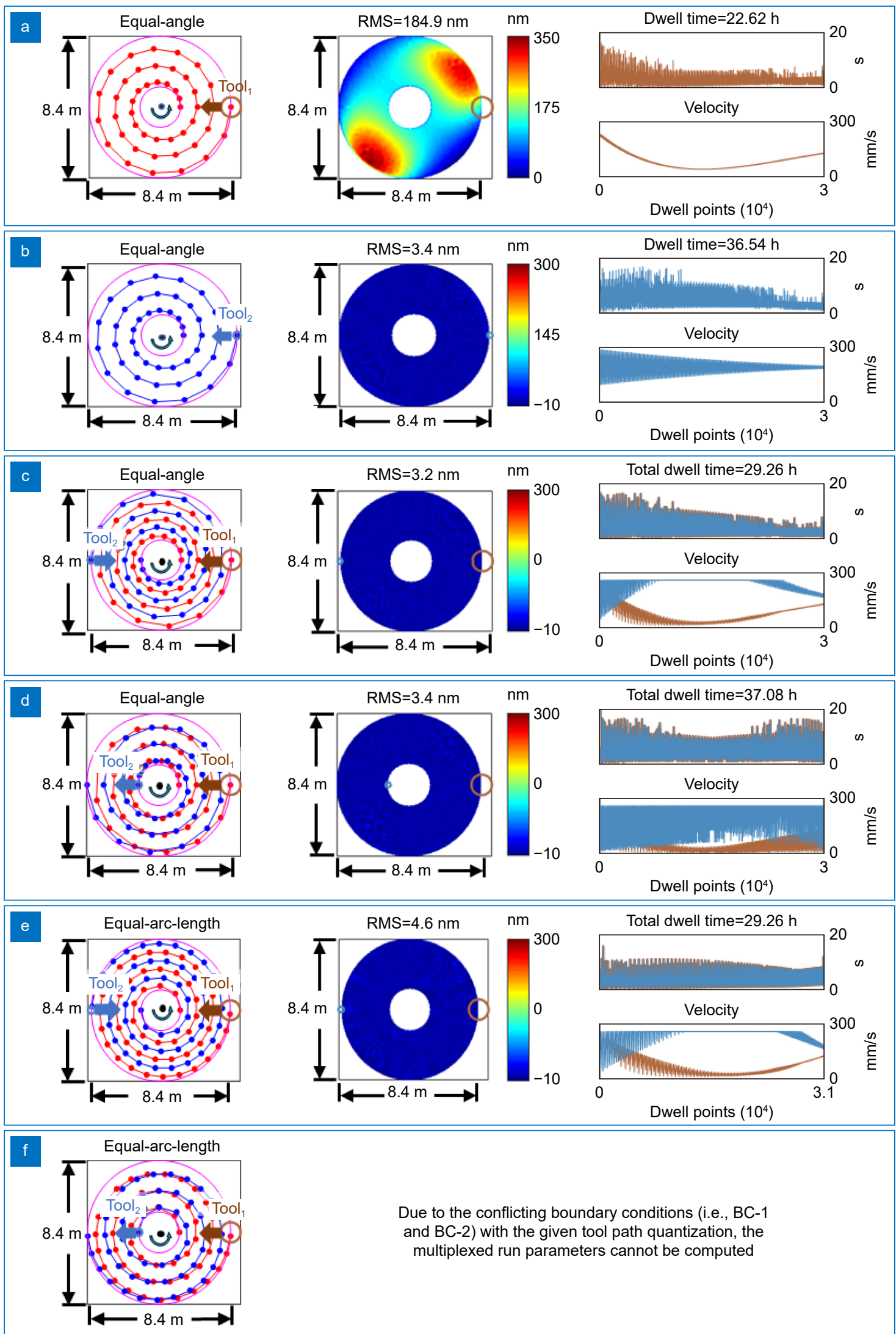

Fig. 3 | The results of simulation: (a-b) two sequential single-tool runs using $\mathrm{Tool}_{1}$ followed by $\mathrm{Tool}_{2}$, (c) in-in feed with equal-angle path, (c) inout feed with equal angle path, (d) in-in feed with equal-arc-length path, and (f) in-out feed with equal-arc-length path. Figure reproduced with permission from ref. ${ }^{6}$, Optical Society of America. 
(1) implies that, to achieve the desired figure in a Clear Aperture (CA), $t(x, y)$ should be optimized on a Dwell Grid (DG) which is larger than the outline perimeter of the CA with the radius of $b(x, y)$. A reliable $t(x, y)$ solution should fulfill three requirements. First, it should be non-negative, since CCOS techniques usually do not have the material adding capability. Second, it should minimize the estimated residual in the CA with the shortest total dwell time to guarantee the fabrication efficiency. Third, the computation time of $t(x, y)$ should be reasonable.

Three categories of dwell time optimization algorithms, namely the matrix-based algorithms ${ }^{30-34}$, the Bayesian algorithm ${ }^{35}$, and the Fourier transform-based algorithm ${ }^{36}$, that partially fulfill these requirements have been attempted. The matrix-based algorithms modeled the deconvolution in an algebraic way, where $t(x, y)$ is solved from an over-determined linear system. Since the matrix is ill-conditioned and rank-deficient, $t(x, y)$ is always unsmooth, unless certain constraints are added $^{32-34,37}$. However, the introduction of constraints makes the linear system much more complicated which cannot be efficiently solved when the measurement scale is large. The Bayesian algorithm ${ }^{35}$ assumes a Poisson distribution of $t(x, y) * b(x, y)$ and a uniform distribution of $z(x, y)$, from which $t(x, y)$ is solved via the Richardson-Lucy algorithm ${ }^{38}$. This algorithm automatically guarantees the non-negativity of $t(x, y)$, however, it contains a regularization hyper-parameter, which is hard to tune in practice. The Fourier transform-based algorithm ${ }^{36}$ is very computationally efficient since deconvolution is transferred to pointwise division in frequency domain by the Fast Fourier Transform (FFT) algorithm as

$$
t(x, y)=F^{-1}\left[\frac{Z_{\mathrm{d}}(u, v)}{B(u, v)}\right],
$$

where " $F^{-1}$ " represents the inverse Fourier transform, $Z_{\mathrm{d}}(u, v)$ is the Fourier transform of the desired removal on the dwell grid, i.e. $z_{\mathrm{d}}(x, y)$, and $B(u, v)$ is the Fourier transform of the TIF $b(x, y)$. However, the close-to-zero frequencies during the division may amplify the noise and a thresholded inverse filtering step is introduced as

$$
t(x, y)=F^{-1}\left[\frac{Z_{\mathrm{d}}(u, v)}{B(u, v ; \gamma)}\right],
$$

where $\gamma$ is the threshold value and

$$
B(u, v ; \gamma)=\left\{\begin{array}{cc}
B(u, v), & \|B(u, v)\|>\gamma \\
\gamma, & \text { otherwise }
\end{array} .\right.
$$

This threshold value again, becomes a hyper-parameter that is usually chosen by trial-and-error.

Based on the Fourier transform-based algorithm, we proposed a Robust Iterative Fourier Transform-based dwell time Algorithm (RIFTA) ${ }^{9}$ that fulfills all the three characteristics of dwell time mentioned above while maintains FFT's high computational efficiency. First, the Nelder-Mead algorithm ${ }^{39}$ was employed to directly search the optimal threshold $\gamma$ for the thresholded inverse filtering. Second, a two-level iterative scheme was proposed to guarantee the non-negativity of the dwell time with the minimal increase in the total dwell time. Third, bicubic resampling was introduced to flexibly adapt the calculated dwell time to any machining intervals in a real CCOS process. The algorithm flow of RIFTA is given in Algorithm 1, which is briefly explained in the followings. Details of RIFTA can be found in ref.'.

Finding the optimal $\boldsymbol{y}$. We define a residual map $z_{\mathrm{r}}$ to be the difference between the desired removal $z_{\mathrm{d}}$ and the removed material $z(x, y)$ as

$$
z_{\mathrm{r}}=z_{\mathrm{d}}-z
$$

The effectiveness of $t(x, y)$ can ben quantitatively evaluated by interrogating the Root Mean Square (RMS) of $z_{\mathrm{r}}$ as $R M S\left[z_{\mathrm{r}}\right]$. The optimal $\gamma$ can be found from an unconstrained optimization problem as

$$
\gamma_{\mathrm{opt}}=\underset{\gamma}{\operatorname{argmin}} \operatorname{RMS}\left[z_{\mathrm{r}}(x, y)\right] .
$$

Substituting Eqs. (7), (9) and (11) to Eq. (12), the optimization objective can be reformulated as

$$
\begin{aligned}
& \gamma_{\text {opt }}= \\
& \underset{\gamma}{\operatorname{argmin} \operatorname{RMS}}\left\{z_{\mathrm{d}}(x, y)-b(x, y) * F^{-1}\left[\frac{Z_{\mathrm{DG}}(u, v)}{B(u, v ; \gamma)}\right]\right\} .
\end{aligned}
$$

It can be observed that the optimization space in Eq. (13) is not smooth due to the thresholding and cropping operations. Its gradients thus cannot be calculated so that any derivative-based optimization algorithms can hardly be applied. In RIFTA, as shown in Line 8 in the RIFTA algorithm in Fig. 4, the Nelder-Mead algorithm is applied to directly search $\gamma_{\text {opt }}$. The initial guess $\gamma_{\text {ini }}$ is obtained as the ratio between RMS $\left[z_{\mathrm{d}}\right]$ and RMS $\left[z_{\mathrm{r}}\right]$ when $\gamma=1$.

Two-level iterative scheme. If the smallest entry of $z_{\mathrm{d}}$ is outside the CA, to ensure the non-negativity of $t$, a constant piston should be added to offset the smallest entry in the DG. This operation, however, will inadvertently increase the total dwell time. Therefore, in RIFTA, 
as shown by Lines $6-16$ in Algorithm (Fig. 4), the inner iterations only depend on the residuals in the CA, i.e $z_{\mathrm{r} \_ \text {ca }}$, to adjust the pistons in the DG. In each iteration, $z_{\mathrm{d}}$ is only offset by a piston of $\min \left(z_{\mathrm{r}_{-} \mathrm{ca}}\right)$, where $\min (\cdot)$ represents the minimum entry in “.”. The negative entries in the calculated $t$, as shown in Line 9, are set as zeros in Line 10 . In this way, $z_{\mathrm{d}}$ is always guaranteed to be adjusted by the smallest pistons during the iterative updates. As shown in Line 15, these inner iterations are performed until the Standard Deviation (STD) of the difference between the current and the previous residuals in the CA is less than the threshold $s t d \_t$ or the maximum number of iteration max_it is reached. The outer iterations are then added to tune the size of the DG, since we

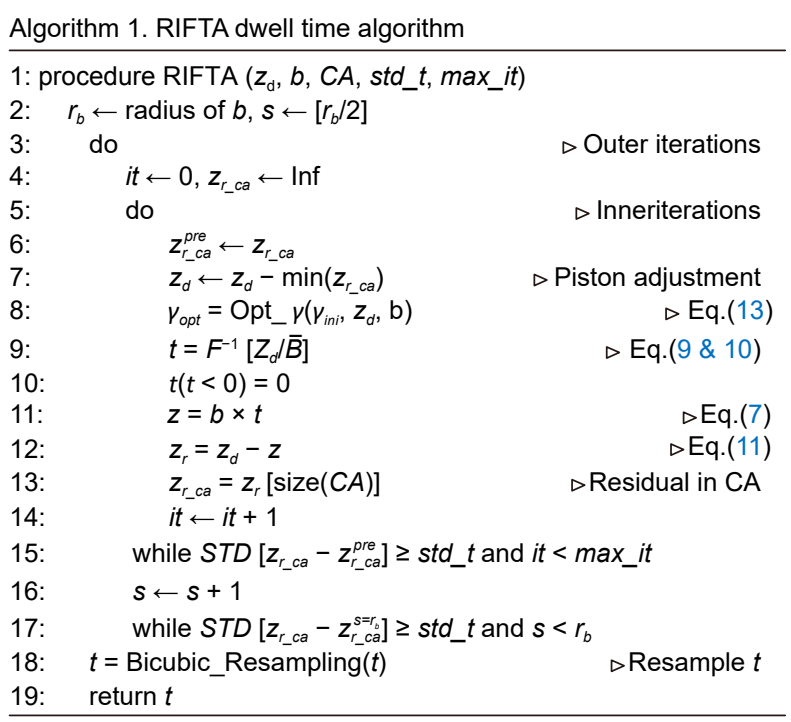

Fig. 4 | Flow of RIFTA dwell time algorithm. found that a much smaller DG is sufficient to obtain the desired $z_{\mathrm{r}_{\mathrm{c}} \mathrm{a}}$ while the total dwell time is significantly reduced. As shown in Algorithm 1, starting from $s=\left\lfloor r_{b} / 2\right\rfloor$, the outer iterations keep searching for the smallest $s$ that fulfills the same stopping criteria as the inner iterations.

Bicubic resampling. To enable the flexibility of having different sampling intervals between metrology and fabrication hardware, we use bicubic resampling to downsample $t$ to any required machining intervals that a CCOS process requires. As shown in Fig. 5, the algorithmic accuracy is not affected by this resampling scheme.

The performance of RIFTA is evaluated on real measurement data shown in Fig. 5(a), where a rectangular flat mirror is measured with $0.12 \mathrm{~mm}$ sampling interval using the sub-aperture stitching interferometry platform ${ }^{40,41}$. The size of the CA is set as $200 \mathrm{~mm} \times 5 \mathrm{~mm}$. The initial figure error in the CA is $167.02 \mathrm{~nm}$ Peak-toValley (PV) and $38.39 \mathrm{~nm}$ RMS. We use a Gaussian TIF with the peak removal rate of $1 \mathrm{~nm} / \mathrm{s}$ and a radius of $5 \mathrm{~mm}$ so that the initial DG size is $210 \mathrm{~mm} \times 15 \mathrm{~mm}$.

Figures $5(b-f)$ shows the different dwell time calculation results. The corresponding estimated residuals in the CA are given in Figs. 5(g-k). Without using RIFTA, as shown in Figs. 5(b) and 5(g), the total dwell time is 3411.32 mins while the residual in the CA remains at $8.44 \mathrm{~nm}$ RMS using $\gamma_{\text {ini }}=1.6709 \times 10^{6}$, which indicates that the calculated dwell time is far from the optimal solution. After $\gamma$ is optimized as $\gamma_{\mathrm{opt}}=1.6650 \times 10^{6}$, the

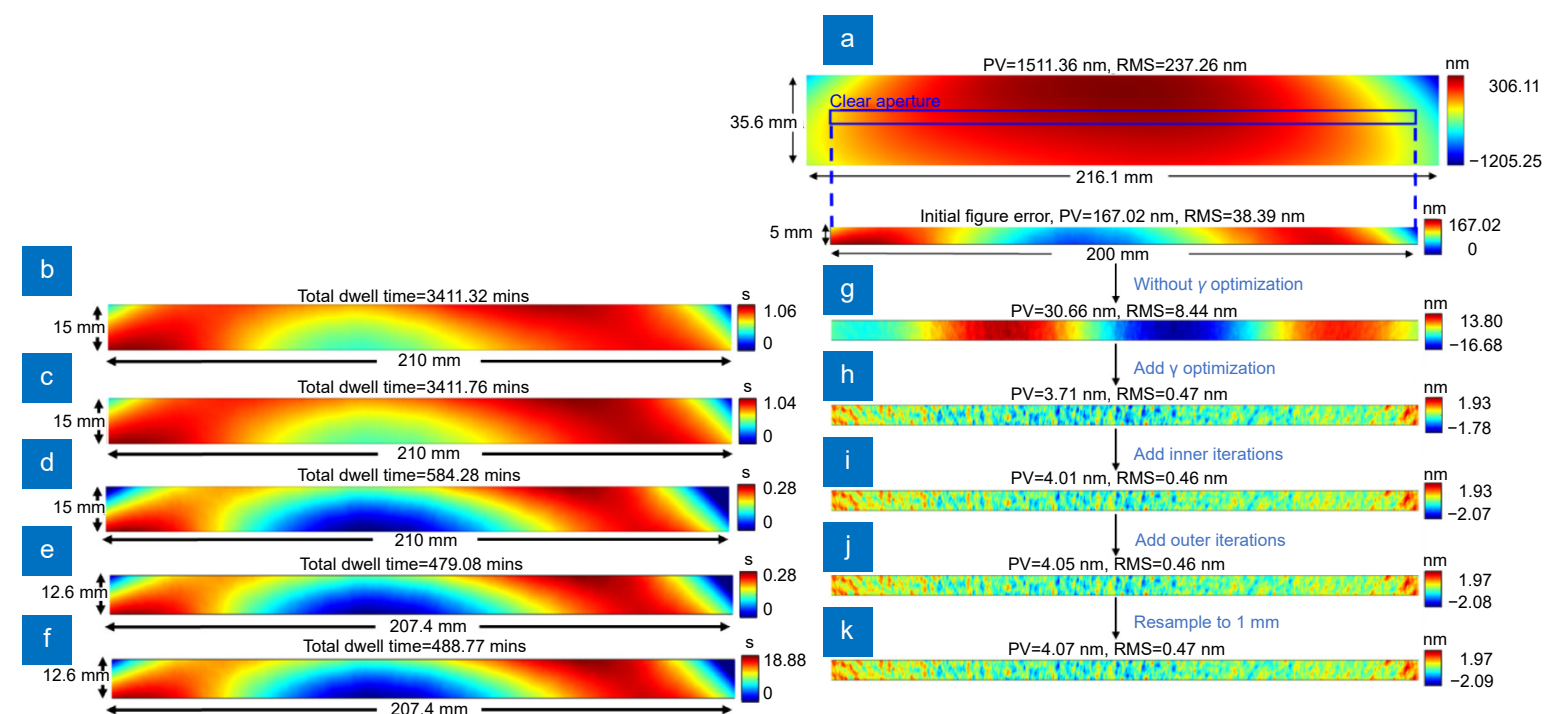

Fig. 5 | (a) Initial surface error map from real measurement, (b-f) dwell time calculation results, and ( $\mathbf{g}-\mathbf{k})$ estimated residuals in the CA without using RIFTA (b, g), adding $\gamma$ optimization $(c, h)$, adding inner iterations $(d, i)$, adding outer iterations (e, j), and resampling to 1 mm (f, $k$ ). 
RMS of the residual in the CA reduces to $0.47 \mathrm{~nm}$ as shown in Fig. 5(h). The total dwell time shown in Fig. 5(c) is similar to that in Fig. 5(b). Afterwards, the inner iterations are employed to shorten the dwell time. Fig. 5(d) shows that the inner iterations bring the total dwell time down to 584.28 mins with an equally good residual in Fig. 5(i). As shown in Figs. 5(e) and 5(j), the dwell time is further reduced to 479.08 mins after the outer iterations for the DG size optimization. The minimized DG size is $207.4 \mathrm{~mm} \times 12.6 \mathrm{~mm}$. The final dwell time, as shown in Fig. 5(f), is obtained by downsampling Fig. 5(e) to $1 \mathrm{~mm}$ sampling interval by bicubic resampling. The final estimated residual in the $\mathrm{CA}$ shown in Fig. 5(k) is not affected by the resampling operation, which proves that RIFTA is an effective dwell time optimization algorithm for CCOS processes.

\section{Very large telescope control system, instrument, and segmentation}

Large, ground-based telescopes dominate modern astronomy. The heritage and lessons learned from these systems create the foundations for future telescopes. In this section, three areas are discussed. The first two, alignment system and cross-dispersion science module, will enable the Large Binocular Telescope (LBT) to produce new and more accurate astronomical observations. The third topic proposes design improvements to telescope mirror segmentation and secondary mirror obscurations created by the support structures.

\section{Large binocular telescope prime focus camera} alignment using laser truss alignment system The Telescope Metrology System measures changes in distance between various points around the primary mirror and retroreflectors fixed to the prime focus camera (LBC), forming a system of laser trusses to determine the "pose" (relative positioning and orientation) of the primary mirror shown in Fig. 6 to LBC prime focus configuration. Each laser truss consists of measurement arms extending from collimators positioned around the primary mirror cell to retroreflectors on the $\mathrm{LBC}$, as shown in Fig. 7. An absolute distance measuring interferometer, the Etalon Absolute Multiline Technology (EAMT), measures laser truss leg lengths with micronlevel accuracy over a 10 meter baseline about once every ten seconds. To determine the pose of the optical elements, an inverse kinematic analysis based on the Stewart-Gough hexapod platform is performed on laser truss lengths. With the pose computed, necessary positioning and rotation corrections may be commanded on the primary mirror to improve collimation and pointing.

The ability of the TMS to measure the relative position and orientation of the primary mirror was validated using incremental motion commanded on the primary mirror, spanning the full range of motion for normal telescope operation. Results for measured $R x$ motion are presented in Fig. 8. The maximum deviation between the calculated pose and the telescope controls was $1.7 \mu \mathrm{m}$ in lateral motion $(X, Y, Z)$ and 0.1 arcsec in rotational motion $(R x, R y, R z)$, which are within the expected range of deviation given the TMS measurement accuracy.

To further verify the validity of the TMS functionality, measured primary mirror position and orientation data were incorporated into an optical model for optical aberration and image quality analysis. For commanded rotations about $X$, or $R x$ motion, Zernike coefficients for

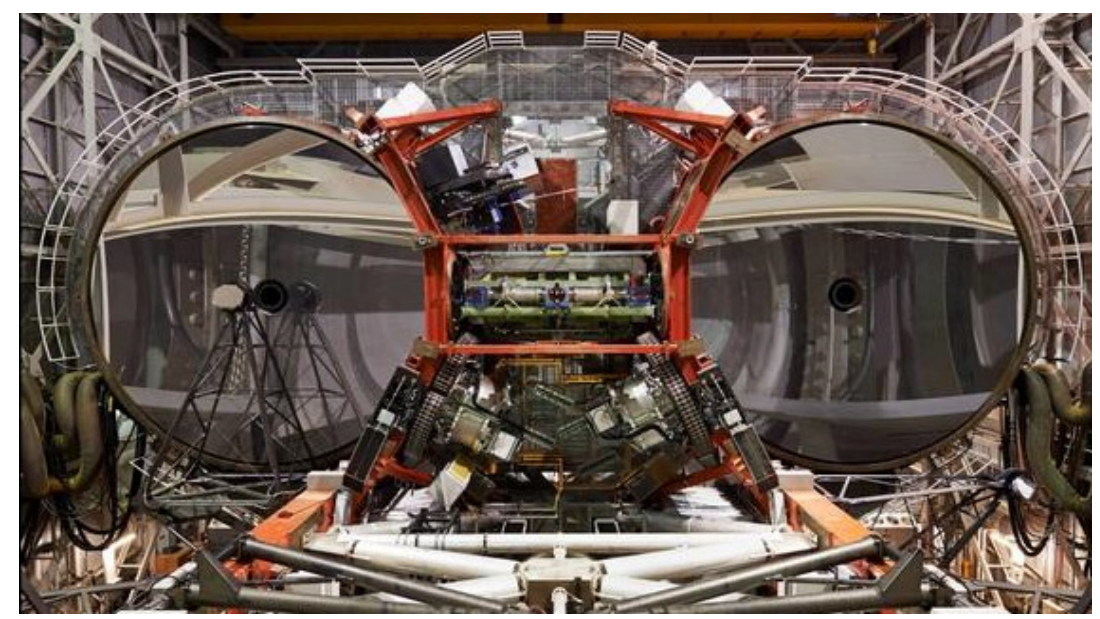

Fig. 6 | Two $8.4 \mathrm{~m}$ in diameter LBT primary mirrors and the science instruments between the two mirrors in the middle of the telescope structure. Figure reproduced from LBTO. 
both horizontal and vertical first-order coma were determined as shown in Fig. 8. Both on-axis and off-axis fields were considered in this analysis, where the field was increased parallel to $Z 7$ horizontal coma. The anticipated field-dependence is exhibited in $Z 7$ magnitude at off-axis field positions. Vertical coma, Z8, exhibits no field-dependence with primary mirror $R x$ rotation as the aberration is oriented perpendicular to the increase in field. Primary functionality of the TMS has been successfully demonstrated at prime focus for monocular mode ${ }^{11}$.

The Telescope Metrology System has begun transitioning from "concept testing" and prototyping on LBT to implementation as an integrated part of the Telescope Control System (TCS $)^{12}$. As such, emphasis has shifted from solely focusing on accuracy to include system reliability and error handling. In its simplest possible configuration, the TMS consists of nine metrology channels

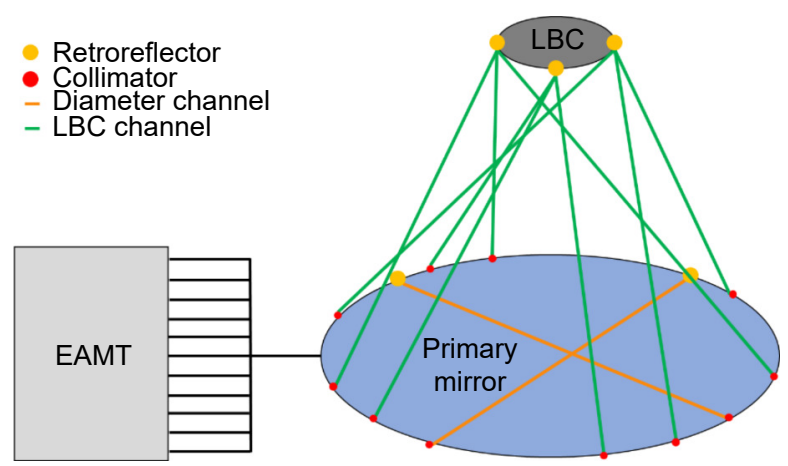

Fig. 7 | Laser truss configuration on the LBT prime focus mode. Laser trusses extend from nine collimators around the primary mirror to three retroreflectors on the prime focus camera, with three collimators aligned to each retroreflector. Additionally, there are two nearly orthogonal channels to monitor the diameter of the mirror. Figure reproduced with permission from ref. ${ }^{11}$, SPIE.

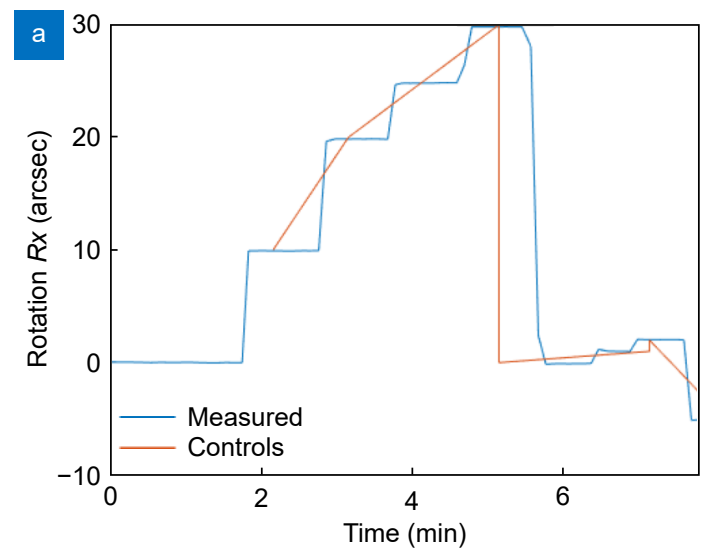

from the right side (DX) primary mirror to three retroreflectors on the LBC red channel. Various system attributes are undergoing refinement during this stage, including: upgrading collimator optics and mount; implementing optical path difference (OPD) corrections to vacuum length; implementing inverse kinematic pose calculations; implementing software error handling; and developing robust and reliable software interaction with the metrology system and telescope.

Achromatic fiber collimators are critically important when using the EAMT system. For monochromatic collimators, the metrology waveband $(1532+/-70 \mathrm{~nm})$ and the alignment waveband $(633 \mathrm{~nm})$ produce very different beam diameters at a distance of 10 meters. To combat this problem, an achromatic collimator was designed to mitigate alignment difficulty. Larger diameter collimators are also being standardized for the TMS, as they improve the beam-walk tolerance and increase the contrast interference signals, improving overall robustness. Besides hardware improvements, enhancements to system calibration in software the OPD of the laser truss path length varies with temperature, pressure, humidity, and $\mathrm{CO}_{2}$ content, with the temperature contribution presenting the dominant effect. Utilizing telemetry from the observatory sensors, Ciddor corrections are applied to optical path length to perform a "vacuum" correction.

To improve the robustness and error handling capabilities of the TMS, the LBTO software group has implemented an TCP/IP (transmission control protocol/internet protocol) software interface, enabling network communication to the EAMT unit via the TCP server ${ }^{12}$. An experimental code has been developed to take

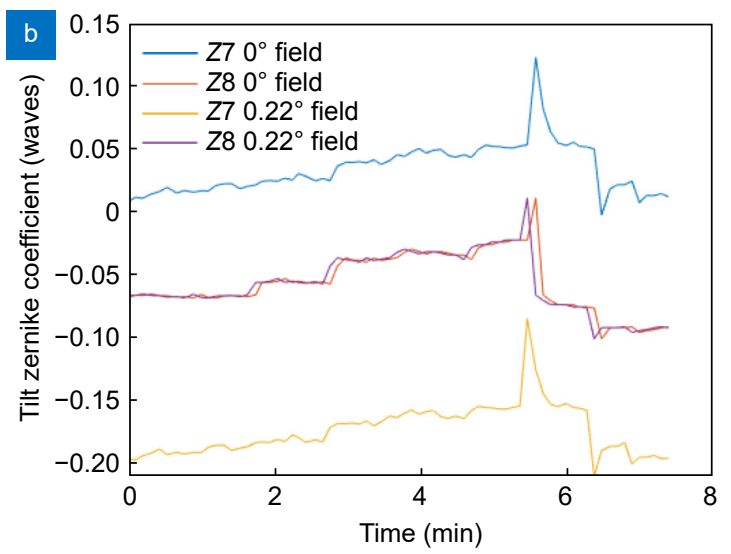

Fig. 8 | (a) The ability of the TMS to measure pose was validated using commanded Rx motion on the primary mirror. (b) Horizontal and vertical first-order coma Zernike coefficients, produced using a perturbed LBT optical model, show the anticipated variability for on-axis and off-axis fields. Figure reproduced with permission from ref. ${ }^{11}$, SPIE. 
measurements, manage interactions between the metrology system and the telescope, and send pose-corrections to the primary mirror. The core functionality of this code is focused on transforming raw channel length measurements to a final "change of pose" vector, which is first adjusted for various telescope instrument offsets, then sent to the TCS as the final correction vector. A schematic outlining the primary functional components of the TMS software is shown in Fig. 9.

First, a pose vector is computed from raw lengths received. Then we subtract the most recent reference pose, the pose measured following the last active-optics cycle using Focal Plane Image Analysis (FPIA). The change of pose is then further processed to combine various terms such as prime focus camera guiding corrections, PSF instrument offsets, as well as primary mirror bending terms, etc. to obtain the final correction to send to TCS.

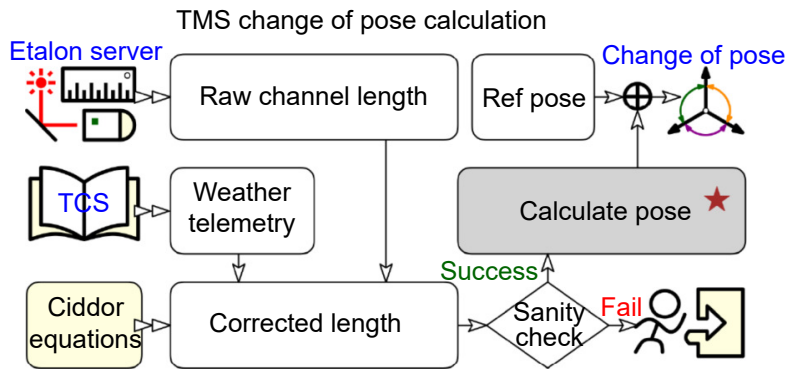

Fig. 9 |Schematic presenting the major steps in transforming raw channel length measurements to a "change of pose" correction vector. The core functionality of the TCP/IP software lies in the shaded, starred box. Figure reproduced with permission from ref. ${ }^{12}$, SPIE.
Following some pending requirements review and onsky commissioning, we are nearly ready to deploy the software for routine use on the LBT.

Currently, the TMS team is working to produce a comprehensive hardware and software metrology package to support ongoing LBC operations. The system has proven successful in passive tests, running in parallel with science observations without sending corrections. Next, the system must complete on-sky commissioning during telescope engineering time. The key benchmarks that the TMS must meet are reliability, accuracy, and operability.

\section{MOBIUS: Cross-dispersion module for Large} Binocular Telescope (LBT)

The MOBIUS (Mask-Oriented Breadboard Implementation for Unscrambling Spectra) is a plug-in module (Fig. 10) which will be placed at the focal plane of LBT to provide cross-dispersion for $\mathrm{LUCI}^{13}$. This module disperses the input slit images in the perpendicular direction to the existing grating to cover zJHK bands simultaneously (Fig. 11) with a single LUCI. As every optical element will be installed inside of current LUCI's slit mask frame, using MOBIUS requires no modifications in current instrument settings. Since MOBIUS consists of two identical cross disperser modules, it will also provide sky subtraction by dithering between source and sky position.

A key optical requirement of MOBIUS is retaining image quality despite the insertion of the module. To verify a

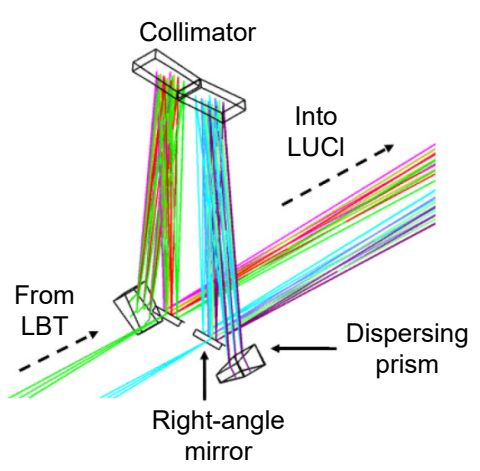

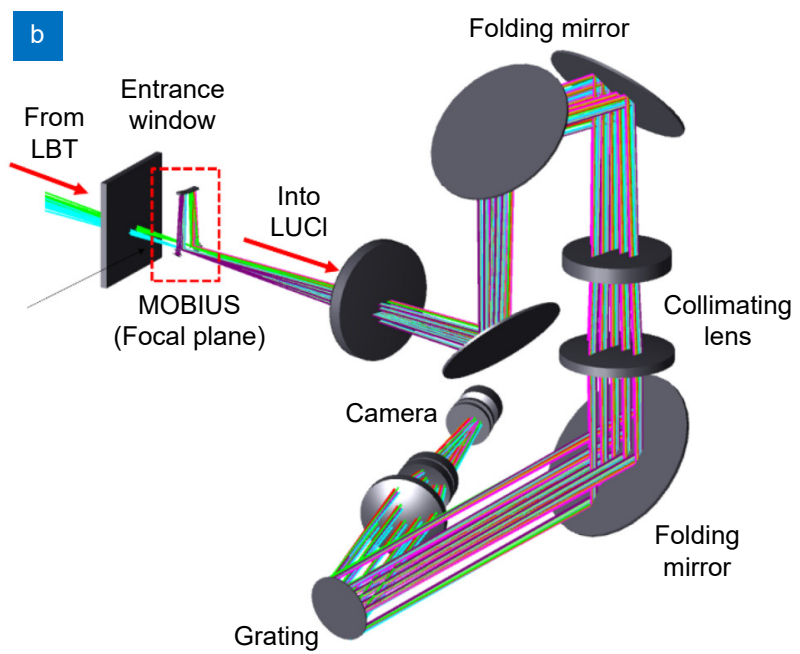

Fig. 10 | (a) Optical layout of MOBIUS. Each MOBIUS module consists of a right-angle mirror, a spherical mirror as collimator, and a dispersing prism which is made of Strontium titanate $\left(\mathrm{SrTiO}_{3}\right)$. The incident beam from LBT will be deflected by the right-angle mirror near the focal plane. (b) As MOBIUS will utilize existing slit mask frame, it requires no modification to current LUCI hardware settings. Figure reproduced with permission from ref. ${ }^{13}$, SPIE. 

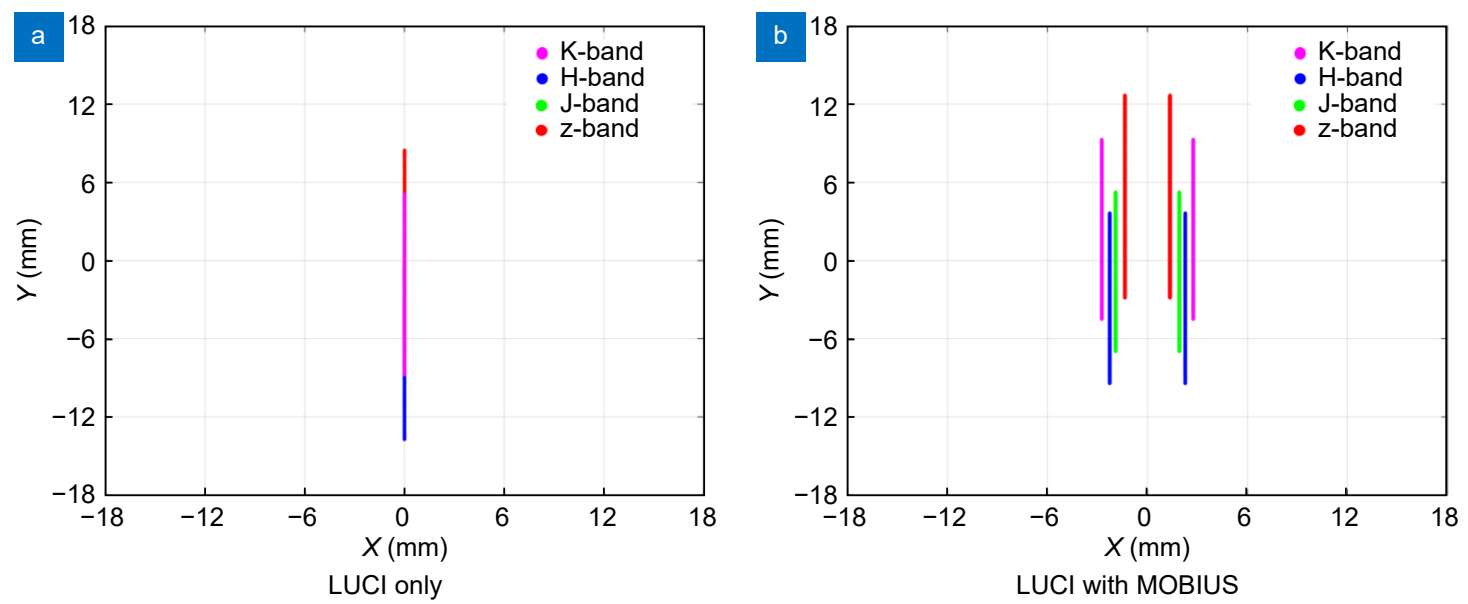

LUCI with MOBIUS

Fig. 11 | (a) Without MOBIUS, ZJHK spectra are overlapped so filters are required to distinguish each band. (b) MOBIUS provides dispersion in the perpendicular direction (shown as in this diagram). As demonstrated, we can simultaneously obtain zJHK spectra simultaneously with 2.3 arcsecond of maximum slit length without overlap. Figure reproduced with permission from ref. ${ }^{13}$, SPIE.

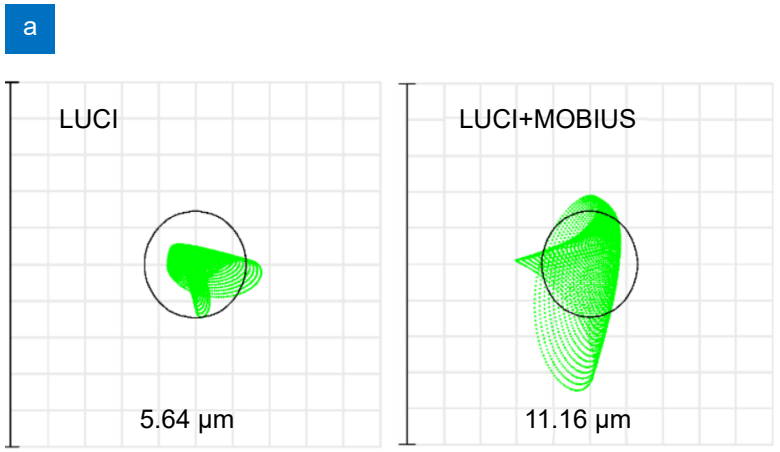

Spot diagram

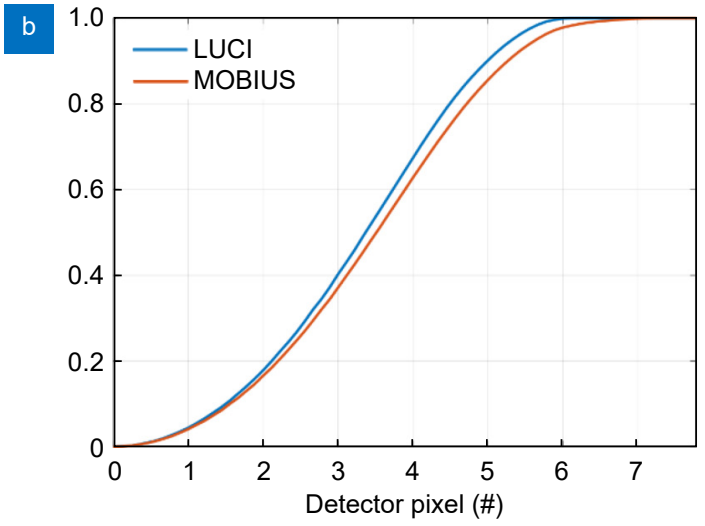

Ensquared energy (K-band)

Fig. 12 | (a) Spot diagram at the LBT focal plane of K-band. The RMS radius of spot size is increased when MOBIUS is introduced. However, considering the expected spot size delivered by the telescope is $150 \mu \mathrm{m}$, the variation caused by MOBIUS is insignificant. (b) Ensquared energy diagram at the LUCl detector plane of K-band. Similarly, the variation induced by MOBIUS is negligible as the pixel size of detector is $18 \mu \mathrm{m}$. Figure reproduced from: (a) ref. ${ }^{13}$, SPIE; (b) ref. ${ }^{42}$, LUCI.

that the image quality is not degraded, we compared the RMS spot radius at the focal plane of the LBT and the ensquared energy at the detector plane (Fig. 12). From simulations, the RMS spot radius increased in every band after MOBIUS was introduced, however, this spot is still smaller than the expected size delivered from the telescope, which is $150 \mu \mathrm{m}$ at focal plane. (Fig. 12(a)) Further, the greatest difference in half width distance for $90 \%$ fraction ensquared energy is also about $2 \mu \mathrm{m}$ which is smaller than a pixel size of detector which is $18 \mu \mathrm{m}$ (Fig. 12(b)). These results demonstrate that the MOBIUS can expand wavelength coverage of a single LUCI while generating negligible variation in optical performance.

Currently the MOBIUS optics elements are fabricated
(Fig. 13(a)) and frame modeling for prototype is done (Fig. 13(b)).

Innovative pinwheel pupil solution for future telescope application

As early as $1941^{43}$, Werenskiold, an amateur astronomer and telescope builder has recorded that curved spiders on secondary mirror could greatly improve the quality of the planetary observation, followed by similar experimental results from Couder (1952) ${ }^{44}$ and Everhart and J. Kantorski (1959) ${ }^{45}$. Until 1984, Richter ${ }^{46}$ provided a detailed Fraunhofer diffraction calculation mathematically and proved that a thin, circular arc obscuration with an angle of $\varphi$ lying in the aperture plane can spread out the flares with the same angle $\varphi$ in the both sides in the 
a

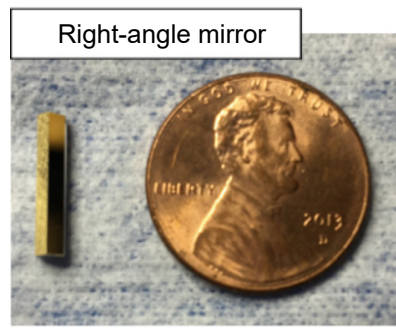

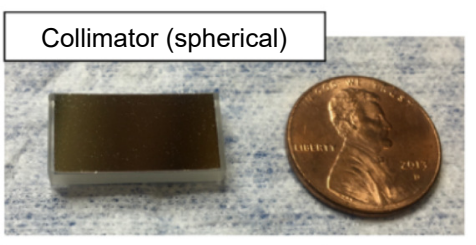

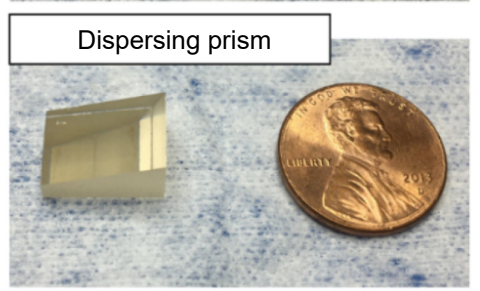

$\mathrm{b}$

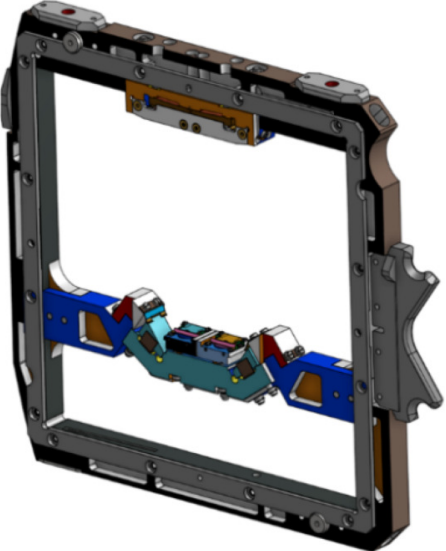

Fig. 13 | (a) Pictures of fabricated MOBIUS optics elements. The size of optics is limited to fit in slit mask frame. (b) Modeling of the MOBIUS-installed frame. To compensate the added weight from MOBIUS, the frame has light-weighted features.

diffraction patterns, shown in Fig. 14.

The previously-mentioned research ${ }^{43-45}$, the secondary mirror spiders from Harvey and Ftaclas $(1995)^{47}$, apodized pupils with spatially varying transmission functions $^{48}$, and the diffraction noise caused by straight edges and gratings of hexagonal segment topology ${ }^{49}$ indicated that the curved-sided segments might be a good alternative for future large telescopes design. Comparing to the high-contrast imaging systems with hexagonal segmented design and delicate instrument for current and future ground- and space-based telescopes ${ }^{50}$, Breckinridge suggested a KISS (Keep It Simple for Space) rule with pinwheel pupil concept for space telescope design ${ }^{51}$. Such concept can reduce the number of optical surfaces, reduce the complexity (active wavefront control or apodized aperture) of the overall system, and then further reduce the cost for future mission. In 2018, Breckinridge and Harvey summarized the previous work, and proposed a pinwheel pupil solution ${ }^{49}$ with digitally simulated results showing without narrow continuous flares and spikes ${ }^{52}$.

In this section, we compare the point spread function (PSF) of a pinwheel pupil (curved edges) and that of a keystone pupil (straight edges) and further illustrate how the curvature of the spokes in the pinwheel pupil plane can impact the PSF in the image plane. The discrete matrix Fourier Transform analysis is performed by Physical Optics Propagation in Python (POPPY) ${ }^{53,54}$, a Python library primarily developed by a team of astronomers at the Space Telescope Science Institute. The investigated pupils are shown in Fig. 15, and they are circular, circular with central obscuration, keystone, Pinwheel 180, Pinwheel 360, and Pinwheel 540 pupil. The numerical num-

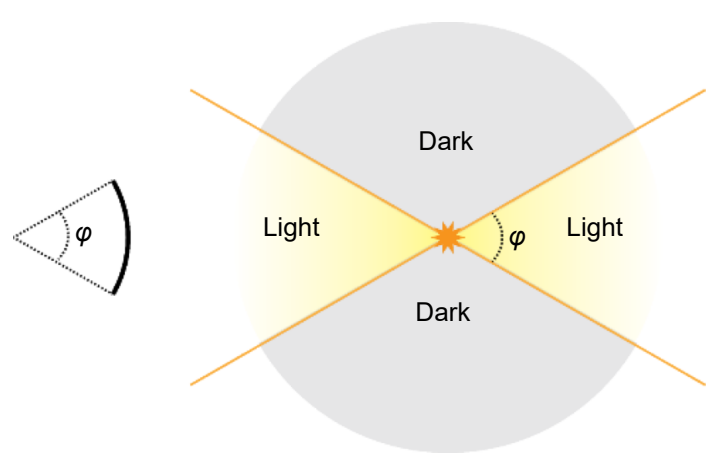

Fig. 14 | A thin, circular arc obscuration with an angle of $\varphi$ lying in the aperture plane is shown on the left. Such obscuration gives rise the "bow-tie/searchlight effect ${ }^{46,49,53}$ in the Fraunhofer diffraction pattern shown on the right.

ber indicated after each pinwheel pupil is the total degree of the curved spokes, in order to meet the bowtie/searchlight effect spreading the light in 360 degree effect ${ }^{46,49,53}$, following the pinwheel pupil prescription from Breckinridge and Harvey ${ }^{52}$. The normalized PSFs are calculated by POPPY. Fig. 16 shows the subtracted PSFs, which means that every normalized PSFs are subtracted by the normalized PSF of the circular aperture (Fig. 15(a)). By doing so, one can easier to observe directly what the footprint can the obscurations, the curved spokes in the pupil plane create in the image plane.

Figure 16(a) shows nothing because the PSF of the circular aperture is subtracted by itself. When the central obscuration is added, Fig. 16(b) illustrated that the energy among the concentric rings. In Fig. 16(c), the segment gaps and straight spokes are added to the aperture plane (Fig. 15(c)), and the consequences are the redistribution of the energy among rings and the straight flares in the PSF. The subtracted PSF of Pinwheel 180 shown in Fig. 16(d) indicates that the curved spokes can redistribute 

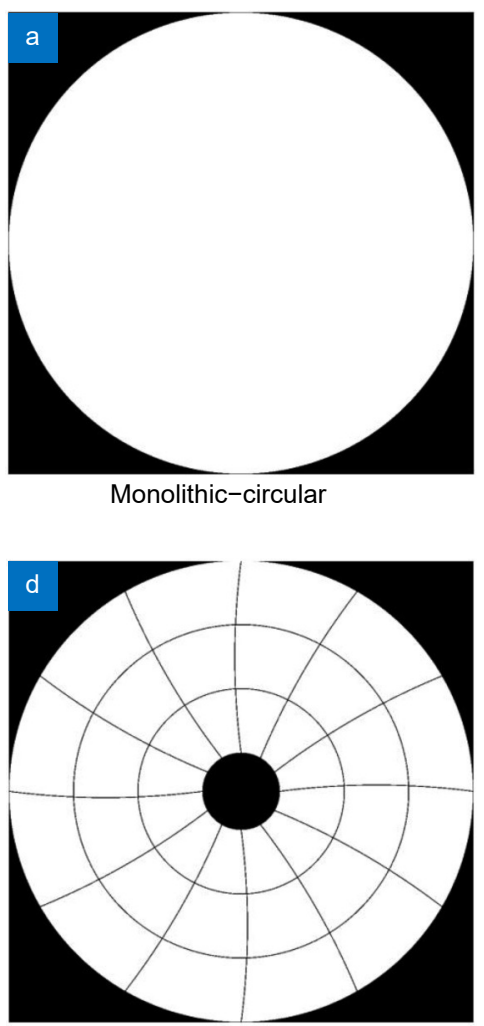

Segmented-pinwheel 180

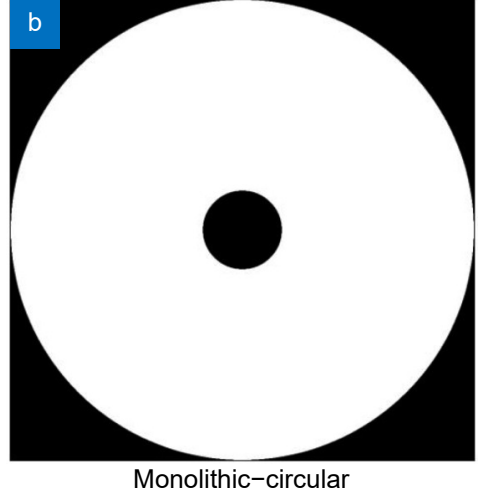

w/central obscuration

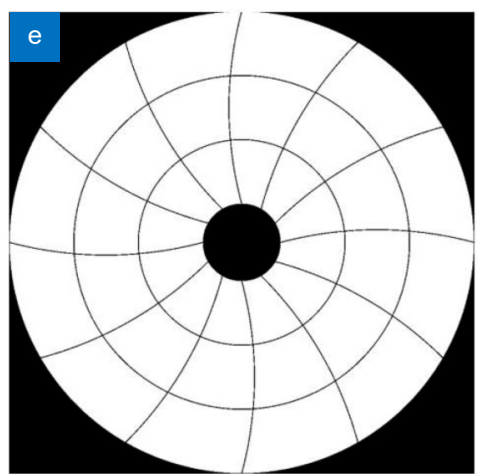

Segmented-pinwheel 360
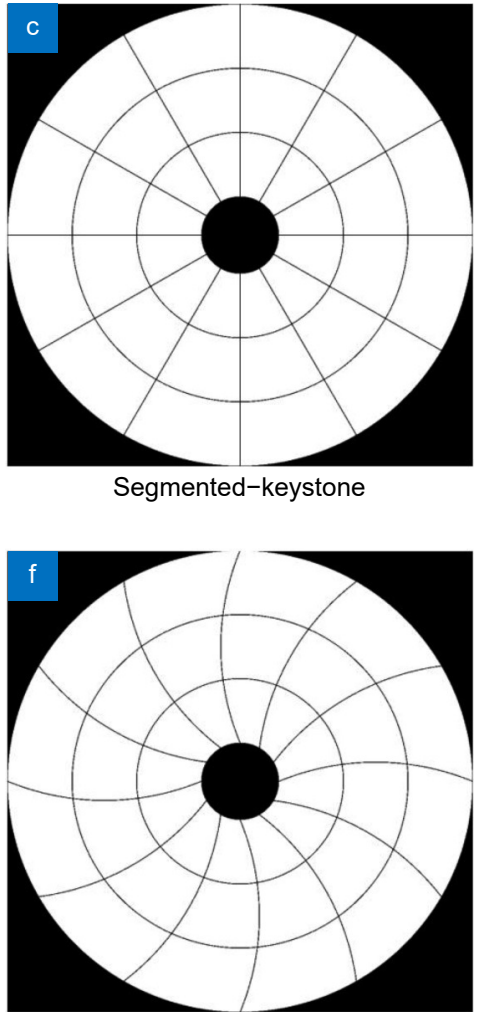

Segmented-pinwheel 540

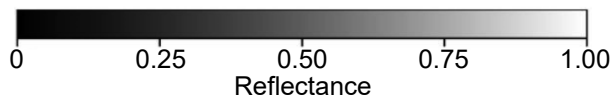

Fig. 15 | Illustration of the segmented pupil topologies studied in this session, and all of them have the same aperture diameter of $12 \mathrm{~m}$ and gap width of $\mathbf{2 0 ~} \mathbf{m m}$. The reflectance of the pupil is color-coded with black and white, the white area is the reflecting surface (mirror), while the black one shows the obscured area. Figure reproduced with permission from ref. ${ }^{14}$, SPIE.

the straight flares along the rings, which is like spreading the beads on the rings, and this is called the "beading effect $^{28}$. More and more curvature of the spokes was added from Pinwheel 180 (Fig. 15(d)), Pinwheel 360 (Fig. 15(e)), and Pinwheel 540 (Fig. 15(f)), and one can observe that the beads are spread out azimuthally following the same direction of the bending of the spokes. Such effect can prevent the straight flares spanning radially across from the center to the outer rings. As a result, the PSF approaches more like an Airy Disk. The tolerance analysis ${ }^{53}$ was presented for the pinwheel pupil. It was illustrated that the pinwheel pupil has low sensitivity in edge error ${ }^{53}$, rotation error, and displacement error.

The pinwheel pupil solution can provide an Airy Disklike PSF with stable performance with the manufacture and alignment error. The fundamental analysis presented in this section is participated to illustrate the advantages of the pinwheel pupil topology. Further investigation including system integration with optimized coronagraphs (e.g., Ruane et al $2018^{55}$ and N'Daiye et al
$2016^{56}$ ) for exoplanet imaging remains to be studied. From the perspective of manufacturing and the KISS rule $^{51}$, the benefit of using a pinwheel pupil provides an innovative solution creating a stable, Airy Disk-like PSF pattern, which can be a great alternative for future space/ground telescope missions.

\section{Future space telescope concepts and enabling technologies}

Astronomers have long desired space telescopes which are free from the many limitations imposed by Earth's atmosphere. To make these future telescopes practical, three new technologies are discussed. First is a discussion of new segmented lens alignment technology designed for exoplanet spectroscopy. The second topic addresses the limited launch capabilities of current rockets by proposing an inflatable primary mirror for terahertz OASIS telescope. As might be imagined, an inflatable mirror has unique metrology requirements. Also, this section introduces a novel space telescope design with cross-dispersed spectroscopy improvements for a far UV 


\section{a}

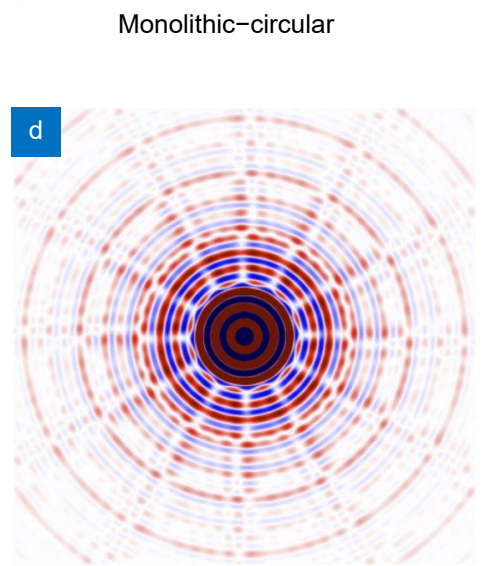

Segmented-pinwheel 180

\section{b}

b

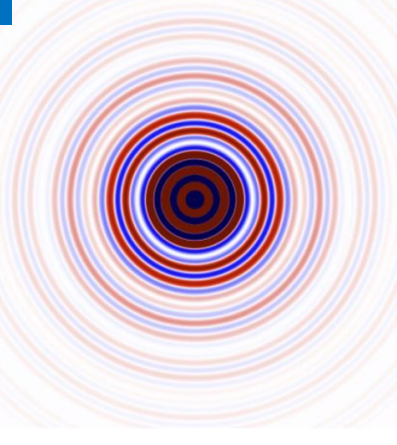

Monolithic-circular w/central obscuration

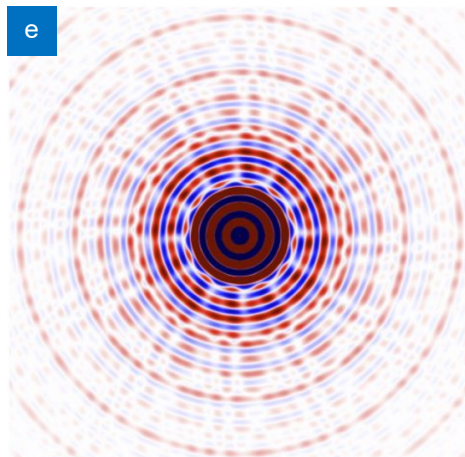

Segmented-pinwheel 360

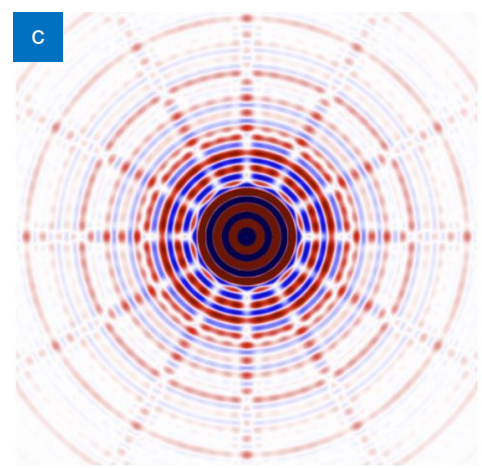

Segmented-keystone

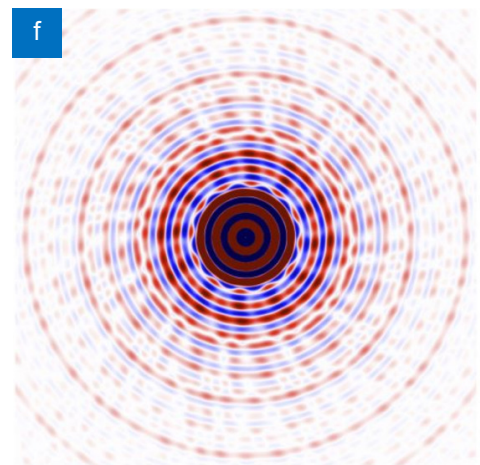

Segmented-pinwheel 540

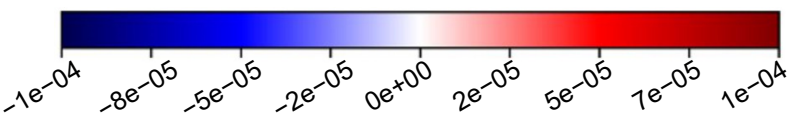

Intensity difference per pixel

Fig. 16 | The normalized PSFs of pupils in Fig. 15, subtracted with that of the circular pupil in Fig. 15(a), i.e. ideal Airy disk function. Note that every PSF is normalized to the peak value before the subtraction is performed.

observation. Finally, this section reviews the CDEEP VVC coronagraph concept including an off-axis primary mirror and a microelectromechanical system (MEMS) deformable mirror (DM). To accurately simulate the incredibly sensitive performance in a relevant space-like environment, a vacuum compatible testbed has been designed to prototype and develop the coronagraph.

\section{Nautilus space observatory for exoplanet} spectroscopy using segmented transmissive optics The Nautilus space observatory for exoplanet spectroscopy utilizes segmented transmissive lenses called MODE lenses as shown in Fig. 17. The alignment tolerances of a segmented MODE lens are on the order of micrometers rather than nanometers. The MODE lens is a novel, lightweight optical component that utilizes both a multi-order diffractive (MOD) lens and a diffractive Fresnel lens (DFL) surface to make a refractive optic that is significantly achromatic and lighter than a typical lens of similar optical power ${ }^{57}$. Its light weight allows for its use in applications such as space observatories. The Nautilus space observatory is one such proposed observatory that would be used for exoplanet spectroscopy and would utilize a segmented MODE lens ${ }^{15}$.

A MODE lens is preferable to a reflective optic in this application because it has much looser alignment tolerances. However, new alignment technologies are still needed to reach these tolerances and meet engineering requirements specific to the MODE lens such as an unobscured aperture for use while bonding the lens segments. To enable the MODE lenses use in such a segmented primary, we have been developing an alignment technology called the Kinematically Engaged Yoke System (KEYS) shown in Fig. $18{ }^{20}$. KEYS utilizes ball-bearings to kinematically contact the MODE lens at the steplike features on the front MOD surface of the lens. This constrains the lens in five degrees of freedom, leaving rotation about the optical axis unconstrained. These ball bearings are attached to set screws, allowing for adjustment of tip, tilt and piston of the lens segments. Two of 

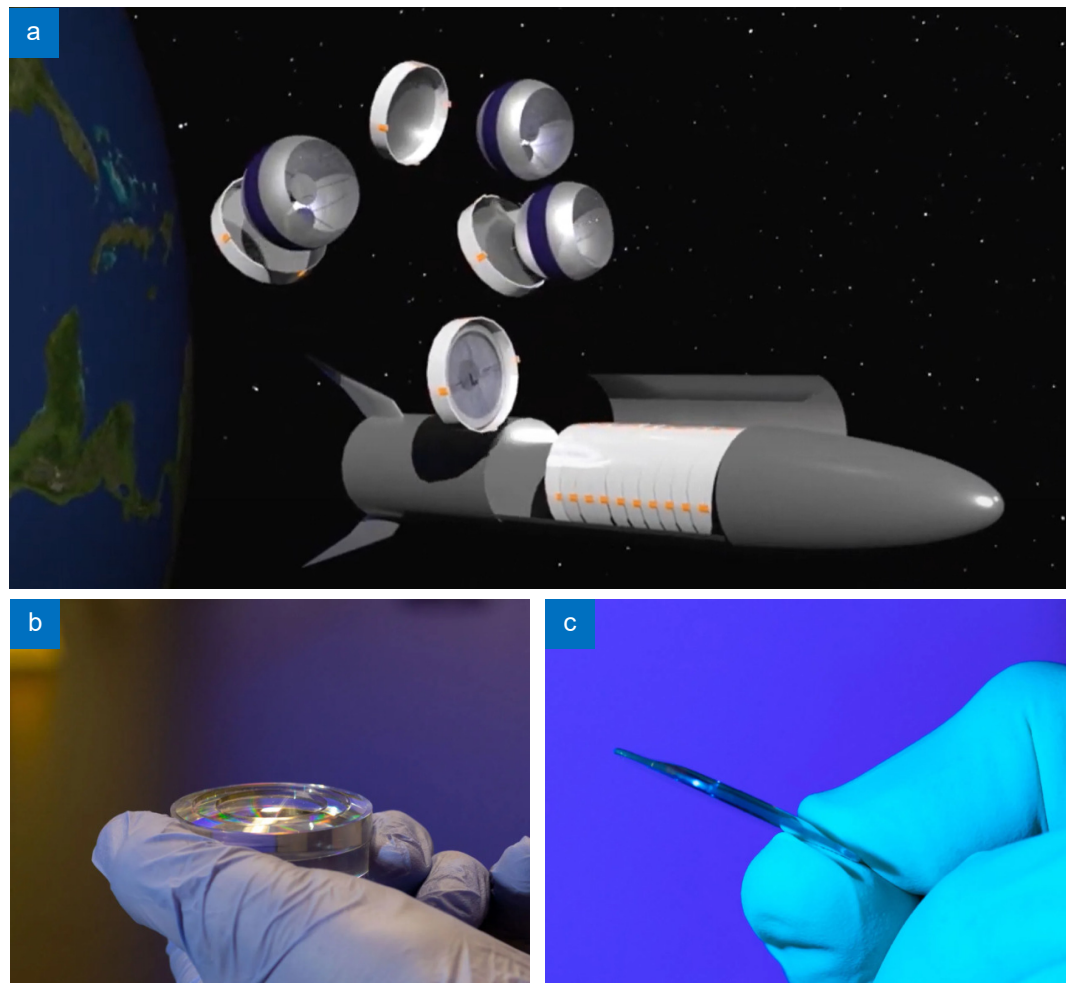

Fig. 17 | (a) Conceptual space deployment image of the Nautilus array in orbit. (b) A color-corrected MODE lens developed by the Nautilus team. (c) A molded Gen4 MODE lens prototype using low-temperature glass.
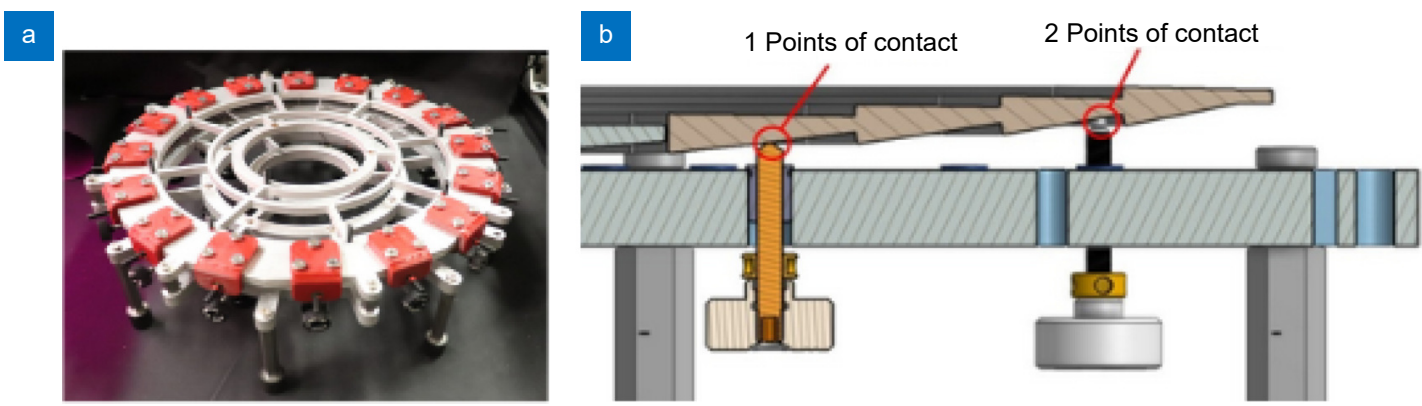

Fig. 18 | (a) The assembled KEYS prototype. (b) A cross-section view showing the contact points of the ball bearings on the step-like features of the MODE lens. Figure reproduced with permission from ref. ${ }^{20}$, SPIE.
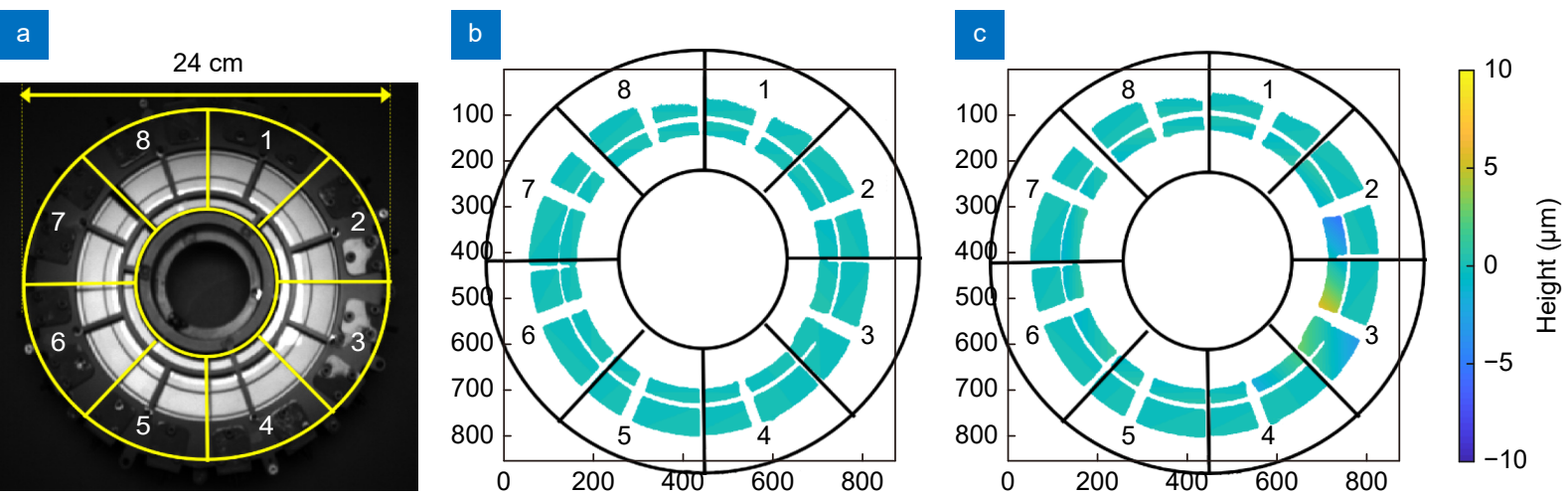

Fig. 19 | The real data from the metrology system. (a) Live view from the camera. (b) All segments are well-aligned against initial co-phasing status. The black line represents the actual size of the single segment. It measures the unobscured area (from the KEYS structure), which is large enough to sense and monitor the misalignment. (c) Segment 3 drifted from the reference position and the measured tilting angle is $0.006^{\circ}$. (The $X$ and $Y$ axis units of (b) and (c) are in pixels.) Figure reproduced with permission from ref. ${ }^{21}$, SPIE. 
the setscrews are mounted on folded flexures which allow for two degrees of freedom of adjustment in the transverse plane.

A prototype KEYS was tested on a $240-\mathrm{mm}$, segmented PMMA MOD lens (no DFL). Our alignment was tested using a deflectometry measurement system and a ZYGO scanning white-light interferometer (SWLI). Using the SWLI, we were able to align segments within 20 microns which is within our tolerances for optical performance and our deflectometry system showed we had a tilt adjustment resolution of $0.006^{\circ}$ as shown in Fig. $19^{21}$.

However, it has become necessary to adjust the lens while testing optical performance in order to be able to compensate for errors made during molding of glass MODE lens segments. In order to accomplish this, we are developing an automatic KEYS that uses actuators rather than manual set screws to make orientation and position adjustments to the lens segments. A stepper motor is coupled to a fine-thread setscrew using a flexible, helical coupling. To achieve finer resolution, those set

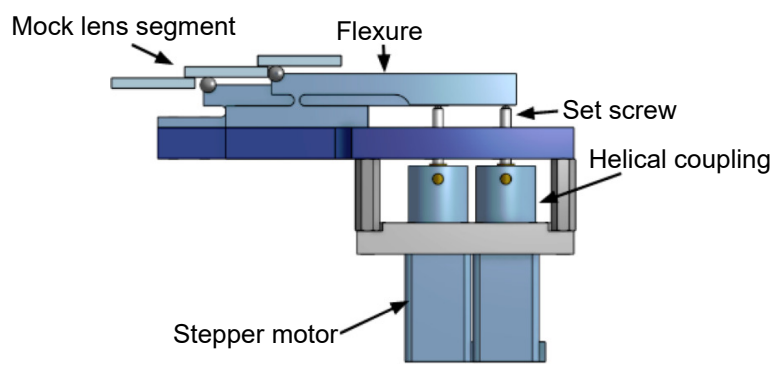

Fig. 20 |Schematic diagram showing a mock-up MODE lens segment (i.e., Mock Lens Segment) mounted on the automatic KEYS adopting computer-controlled stepper motor actuators to make closed-loop orientation and position adjustments (i.e., alignment and co-phasing) to the mock-up MODE lens segments. screws push on lever flexures that have ball bearings adhered to them in order to kinematically contact the lens segment as shown in Fig. 20.

\section{Inflatable reflector metrology for OASIS terahertz space telescope}

The Orbiting Astronomical Satellite for Investigating Stellar Systems (OASIS) is a 20-meter class space observatory that uses an inflatable membrane primary as shown in Fig. 21. Some challenges to measuring the specular concave surface include its large size, manufacturing variations such as wrinkles, and intentional but possibly large variations from annealing ${ }^{58}$.

Deflectometry is well-posed to meet these metrology challenges because it is able to measure specular surfaces and can be reconfigured to measure a vast range of surface slopes, such as those produced at various inflation pressures $^{59,60}$. The principal challenge to deflectometry in achieving global accuracy has historically been geometric calibration, but testing at a long distance lends the ability to achieve the required $\lambda / 8 \approx 67.5 \mu \mathrm{m}$ accuracy by using a portable deflectometer head that is calibrated offsite and aligned to the test surface in-situ ${ }^{24}$.

Measurement uncertainty in the calibration of the system components, such the camera, test surface, and spatial light source, $\vec{r}_{\mathrm{c}}, \overrightarrow{r_{\mathrm{m}}}$, and $\overrightarrow{r_{\mathrm{s}}}$, introduces different shape errors. Defining the direction $\widehat{z}$ as parallel to optical axis of the nominally rotationally symmetric surface, axial miscalibrations along $\widehat{z}$ produces radially symmetric error such as excess defocus or spherical terms, while lateral miscalibrations in the $\hat{x}$ or $\hat{y}$ directions embed excess astigmatism or higher order asymmetric error into the obtained measurement shape ${ }^{61}$.

Exploring deflectometry for a 1-meter OASIS primary

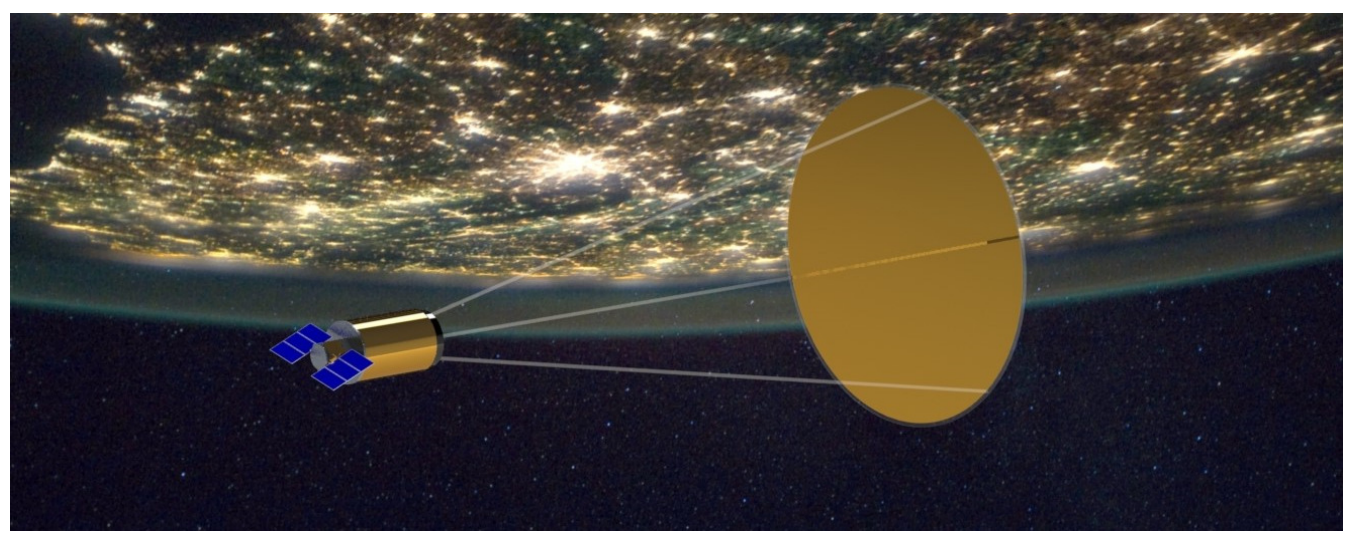

Fig. 21 | Conceptual rendered image of the OASIS space observatory with a 20-metrer diameter inflatable primary aperture (yellow disklike membrane on the right side of the image). 
antenna model, we show that the required calibration error by an external measurement device is relatively relaxed in the axial direction. At a measurement distance approximately equal to the radius of curvature $(\sim 4 \mathrm{~m})$ in the inflated $\mathrm{f} / 2.2$ configuration, an uncertainty $\varepsilon_{z}$ within a bilateral $3.5 \mathrm{~mm}$ envelope is sufficient to produce the desired $\lambda / 8$ measurement accuracy specification, shown in Fig. 22. Because the scale of the optical test setup (meters) dwarfs the uncertainty of the calibration measurement devices (millimeters), excess rotationally symmetric induced errors are not as nefarious in a long-dis- tance deflectometer as they have been in smaller testing configurations.

As for errors induced by lateral calibration uncertainty, induced asymmetric shape can be removed using the N-Rotations methodology, familiar in the absolute interferometry literature ${ }^{62}$. The central thesis of these methods is that a measurement consists of inherent surface shape and spurious shape imbued by systematic calibration error. While the true surface shape rotates if the optic is physically clocked, the systematic calibration error does not. If measurements of a clocked optic are

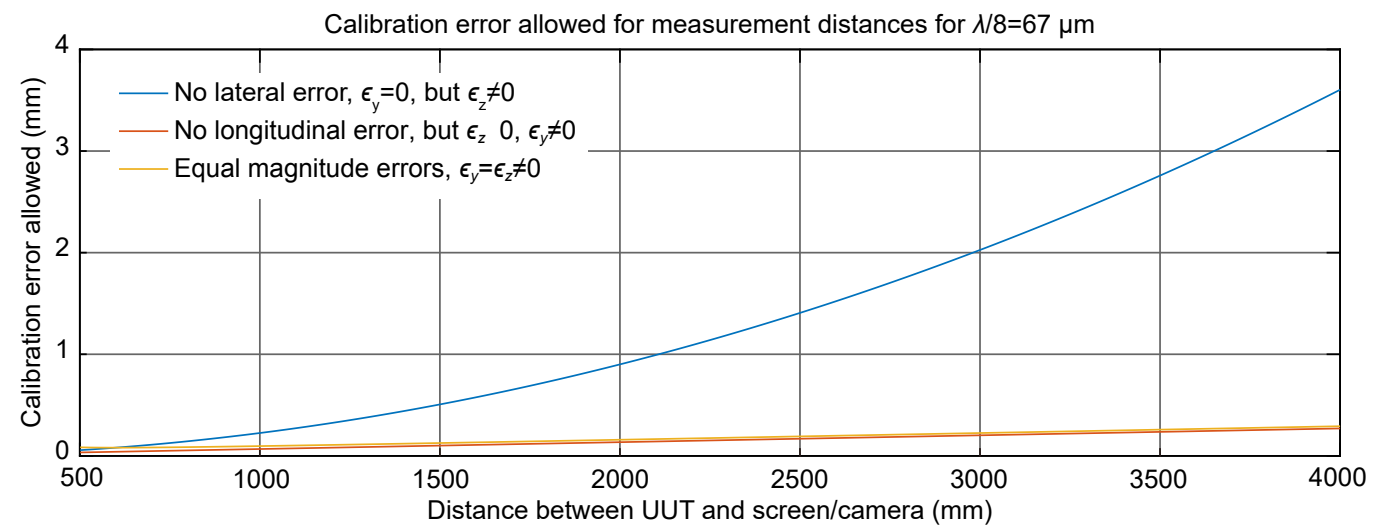

Fig. 22 | A coarse tool such as a laser distance gauge can easily measure the longitudinal distance within 2 millimeters. The shape error sensitivity is vastly dominated by the lateral calibration errors $\varepsilon_{y}$ or $\varepsilon_{x}$, which require 300 microns of accuracy to meet the global measurement requirement. Since the compact deflectometer head is portable and quasi-kinematically mounted, this can easily be achieved in an offsite coordinate measuring machine.
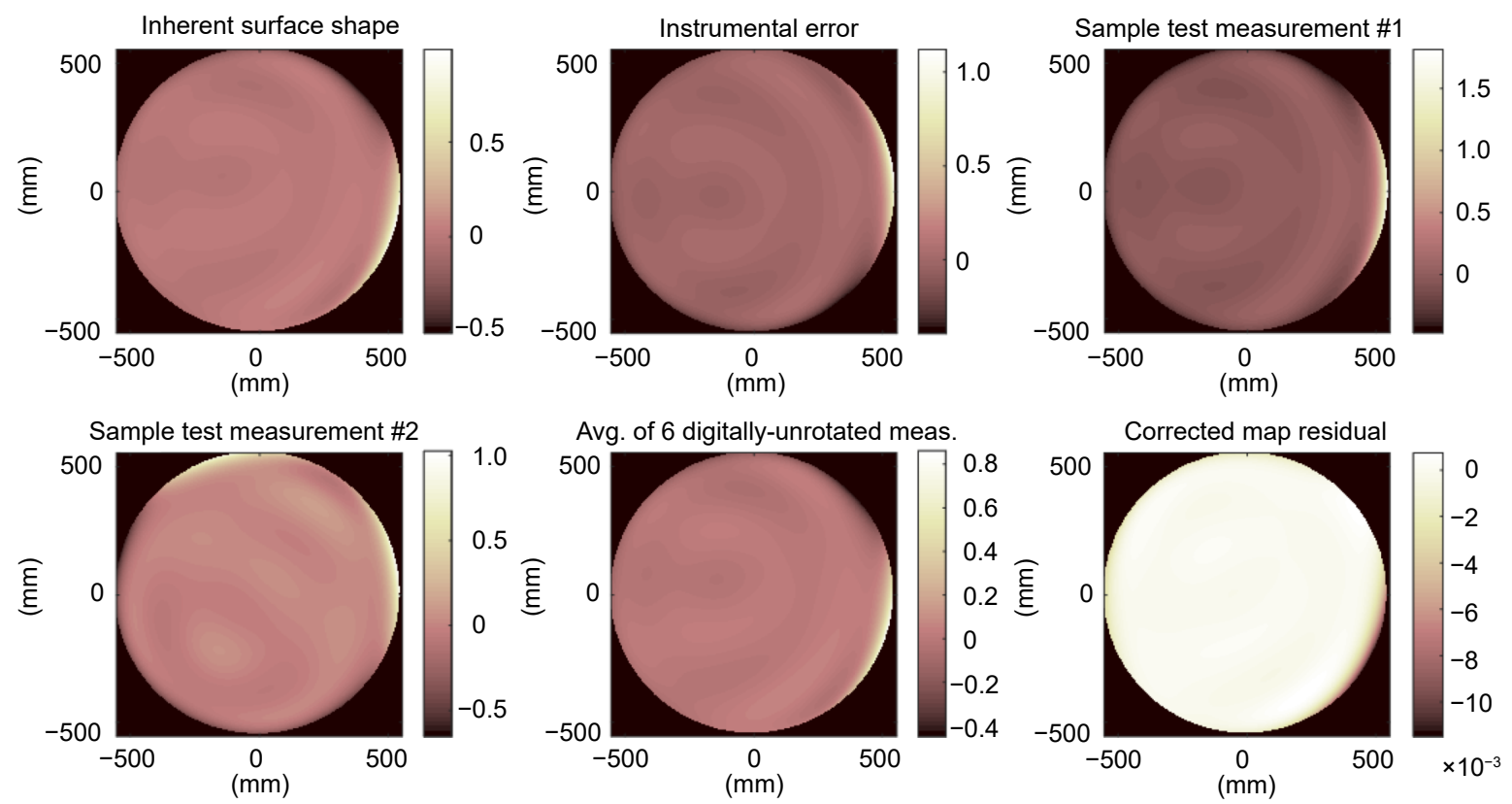

Fig. 23 | A simulation showing inherent surface shape and instrument test error is provided. Sample test measurement 1 and 2 differ in that a rotated surface shape was added to the static instrumental error map to produce them. 'Unrotating' each of the six maps to the original unclocked positions produces an average that is remarkably similar to the true inherent surface shape. Taking the difference between this average and underlying inherent surface shape yields an error of less than $0.5 \%$ of the systematic error originating from the measuring instrument. 
digitally unrotated and then averaged, rotationally asymmetric terms can be precisely cancelled out. Fig. 23 demonstrates this concept by simulating an inherent test shape and systematic error (in $\mathrm{mm}$ ), measurements of the clocked optic, and the asymmetric term removal result. A clocking uncertainty of 0.5 degrees is added into the 6-rotation simulation to demonstrate its efficacy even with physical rotation stages.

Combined, the OASIS deflectometer concept is less susceptible to induced rotationally symmetric errors because of its long-distance testing configuration and its ability to rotate coaxially about the antenna's optical axis. The 1-meter prototype configuration (Fig. 24) is used in scaling studies of the inflatable membrane architecture over a large range of pressures and up to the $20 \mathrm{~m}$ scale version.

\section{Long-slit cross-dispersion spectroscopy for} Hyperion FUV spectroscopy space telescope Hyperion space mission (Fig. 25) targets the observation of Far Ultraviolet (FUV) spectral range where its investigation reveals the secret of the first stage of the star formation ${ }^{63-65}$. Due to the spectrum complexity and population within the interesting FUV range (1405 $\AA-1645$ $\AA, 1 \AA=10^{-10} \mathrm{~m}$ ), the high spectral resolution spectroscopy with a wide field of view is an essential specification. The astronomer demand drives the optical design goal to achieve 5 arcsec spatial resolution, $10 \mathrm{arcmin}$ instantaneous field of view, and greater than 30000 spectral resolution $(R=\lambda / \Delta \lambda)$.

In order to meet the optical performance requirements, we adopted the extreme aspect ratio long-slit (240 aspect ratio, $10 \operatorname{arcmin} \times 2.5$ arcsec) on the RitcheyChretién telescope. The hyperbolic mirrors for primary and secondary are designed to have the $2,400 \mathrm{~mm}$ effective focal length, and it passes the F/ 6 beam to spectroscopic apparatus ${ }^{25,26}$. The extreme aspect ratio of the long-slit and the high spectral resolution requirement $(R>30,000)$ at the FUV spectral range makes the Hyperion's optical design unique. The conventional spectrometer optical layout (off-axis collimator-grating-imaging optics) induces the significant aberration, especially when the long slit has to be installed into the high-resolution spectroscopy. We introduced the novel on-axis
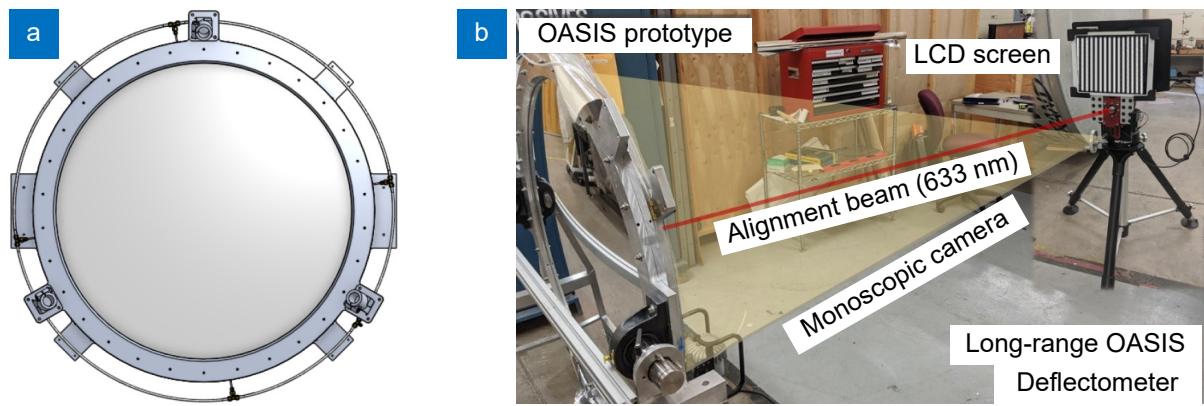

Fig. 24 | (a) Front view of the inflatable 1-meter OASIS primary aperture prototype solid model. (b) The deflectometer head is mounted onto a telescope mount and features a typical machine vision camera, a high-quality LCD display, and a laser for alignment with the test mirror's optical axis. The deflectometry assembly (LCD screen, laser, and camera) was affixed to an OPTRON telescope mount with 3 DOFs so alignment could be achieved between the mechanical axis of the OASIS 1-meter prototype and the calibrated center point of the deflectometer.

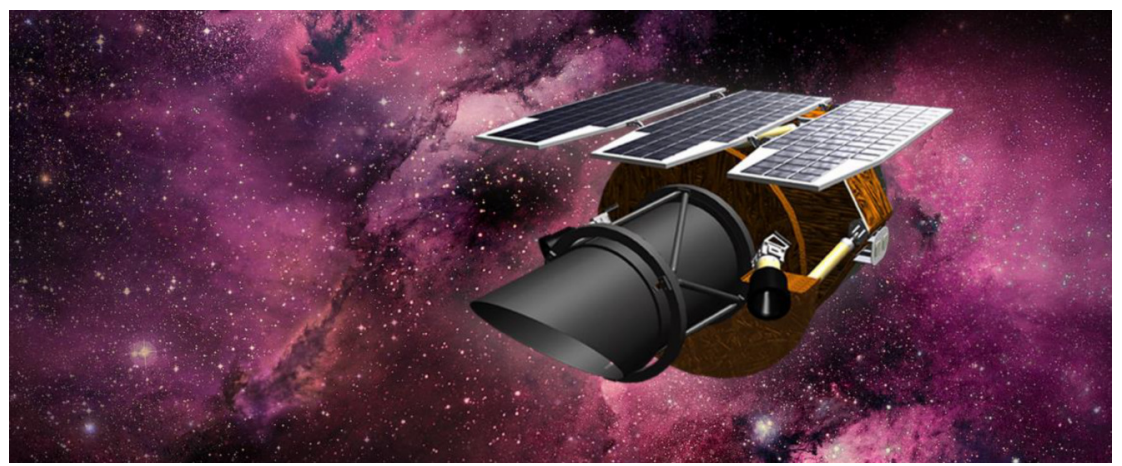

Fig. 25 | The rendering image of the UV space telescope Hyperion examining the fuel for star formation by probing the nature, extent, and state of $\mathbf{H}_{2}$ at the crucial atomic-to-molecular interstellar medium boundary layer ${ }^{25,26}$. Figure reproduced with permission from ref. ${ }^{25}$, SPIE. 
layout applying the collimation mirror on-axis through the center hole of the first grating (échelle grating), and the freeform imaging mirrors are embodied (Fig. 26). The elliptical center hole on the échelle grating is designed to have zero photon loss by matching the secondary mirror's obstacle as shown in Fig. 26(a).

The on-axis layout of the primary mirror - secondary mirror - tertiary mirror - first grating (échelle) gives the minimized the field aberration along the slit length ${ }^{66,67}$. Moreover, the in-plane layout of the first grating (échelle) - cross dispersion grating - two freeform mirror - detector allows us to estimate and handle the aberration easily. Since the cross dispersion divergence angle after both gratings is large, the formation of the two free- form mirrors and surfaces are optimized based on the previously reported Type-4 design form approach ${ }^{68}$. Once the optical design is selected, the diffractive performance is evaluated for the entire spectral range. Even though the geometric optical optimization carefully selects the grating diffraction order and line density, it doesn't confirm the spectral efficiency. Furthermore, because the high-order échelle grating has a delicate spectral sensitivity, the diffraction efficiency simulation is an essential procedure. Based on the electromagnetic wave propagation simulation, both gratings' blaze angles are determined to have the best efficiency at the targeting order as shown in Fig. 27.

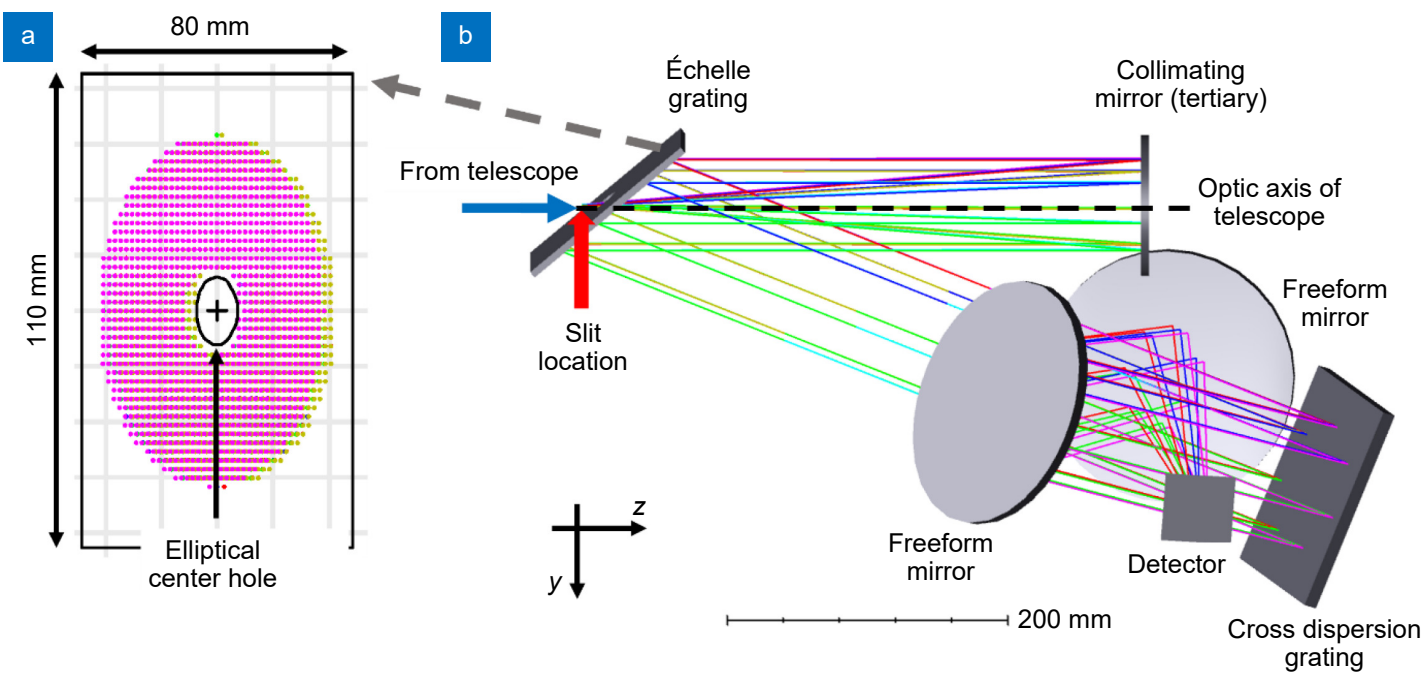

Fig. 26 | The cross-dispersion spectroscopy optics layout (b) and beam footprint on the échelle grating plane (a). Figure reproduced with permission from ref. ${ }^{25}$, SPIE.
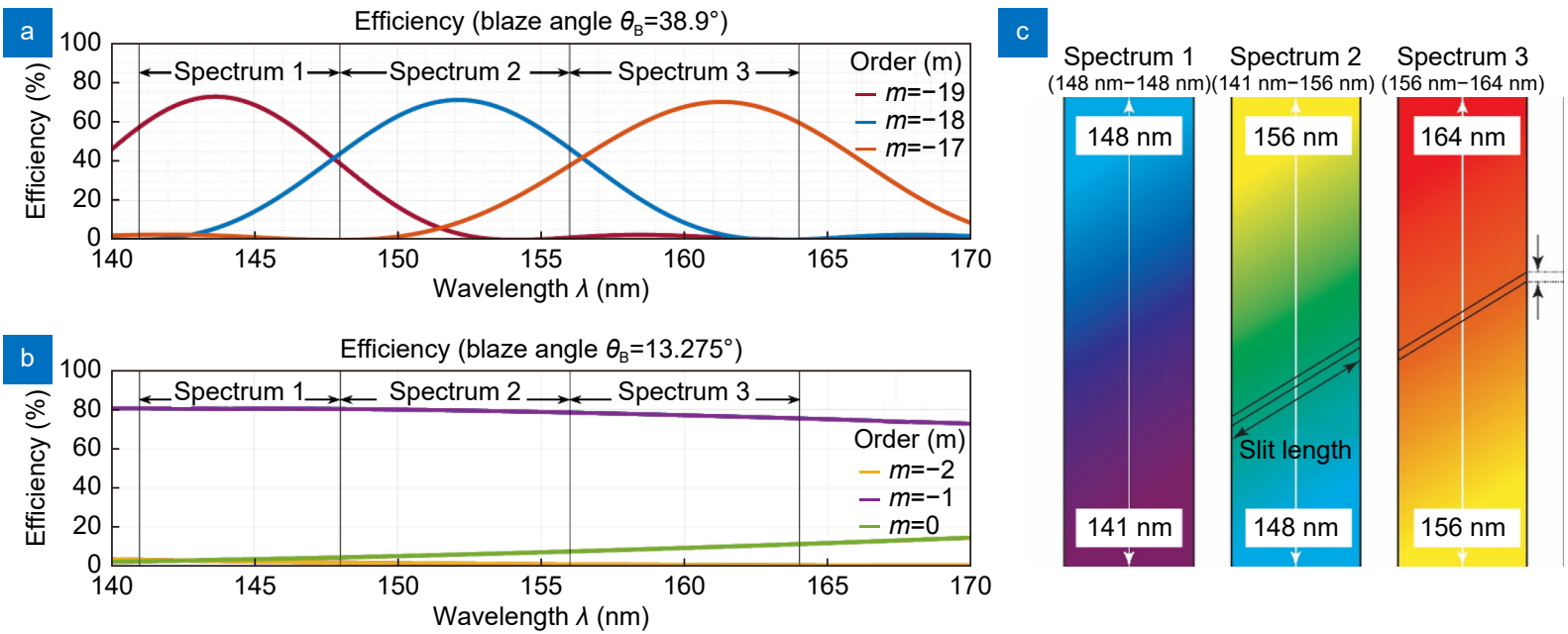

Fig. 27 | The diffraction efficiency calculation results for (a) échelle grating and (b) cross dispersion and (c) spectrum distribution on the $52 \mathrm{~mm} \times$ $52 \mathrm{~mm}$ sensor plane. The three orders of the échelle grating are overlapped, but those are separated by cross dispersion horizontally on the sensor. The high-order of échelle grating dispersive the spectrum rapidly, and it implements the $R>30000$. Figure reproduced with permission from ref. ${ }^{25}$, SPIE. 
Coronagraphic debris exoplanet exploring pioneer (CDEEP) for SmallSat space coronagraph

The Coronagraphic Debris and Exoplanet Exploring Pioneer (CDEEP) is a SmallSat mission concept developed by the UArizona Space Astrophysics Laboratory in collaboration with the Large Optics Fabrication \& Testing group. As a coronagraphic instrument which blocks onaxis starlight to allow detection of dim circumstellar debris disks, CDEEP is proposed to be a monolithic silicon carbide $(\mathrm{SiC})$ three-mirror telescope and coronagraph instrument featuring a $34.9 \mathrm{~cm}$ primary mirror as shown in Fig. 28. This SmallSat design also serves as a technical pathfinder for future space coronagraph telescopes utilizing even larger monolithic mirror apertures $^{69}$. CDEEP leverages heritage from the Deformable Mirror Demonstration Mission (DeMI) ${ }^{70-71}$, the PICTURE series balloon ${ }^{72}$ and sounding rocket missions $^{73-75}$, EXCEDE laboratory testing ${ }^{76,77}, \mathrm{SiC}$ optics $^{78,79}$ and the vast theoretical and experimental work conducted toward the development of VVC technology ${ }^{80-83}$. The off-axis fore-optics eliminate problematic diffraction features from secondary mirror supports that would otherwise make high-contrast imaging more challenging. The fast Cassegrain objective feeds light to the fast-steering mirror (FSM) for pointing correction, and then to the DM for static and dynamic aberration correction. The corrected light is then imaged onto the VVC which rejects on-axis starlight while passing light from a debris disk and/or planet unperturbed. The rejected starlight is vignetted by a reflective Lyot stop and fed to the low-order wavefront sensor, while the light from the target of interest is passed to the science camera suite ${ }^{84}$. With post-processing, the CDEEP coronagraph will achieve contrast levels of $10^{-8}$, enabling
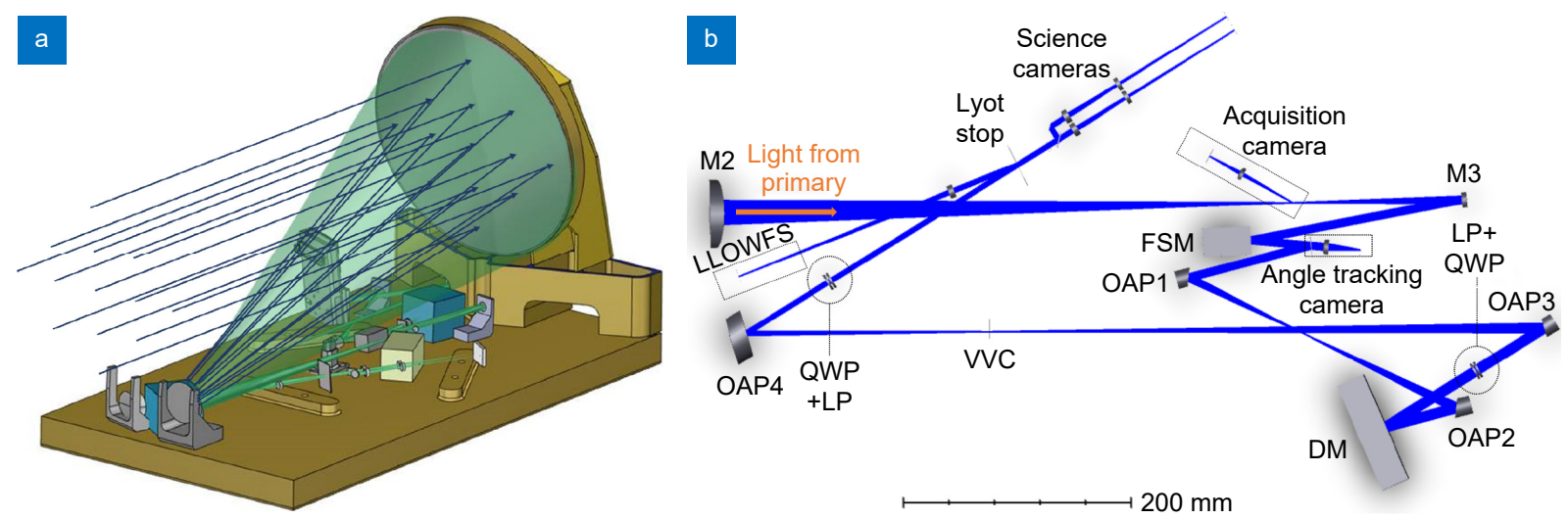

Fig. 28 | (a) Optomechanical rendering of the CDEEP optical configuration showing the unobscured SiC telescope and integrated optical bench. (b) Raytrace of the coronagraph optical train beginning at the secondary mirror M2. Figure reproduced with permission from ref. ${ }^{27}$, SPIE.

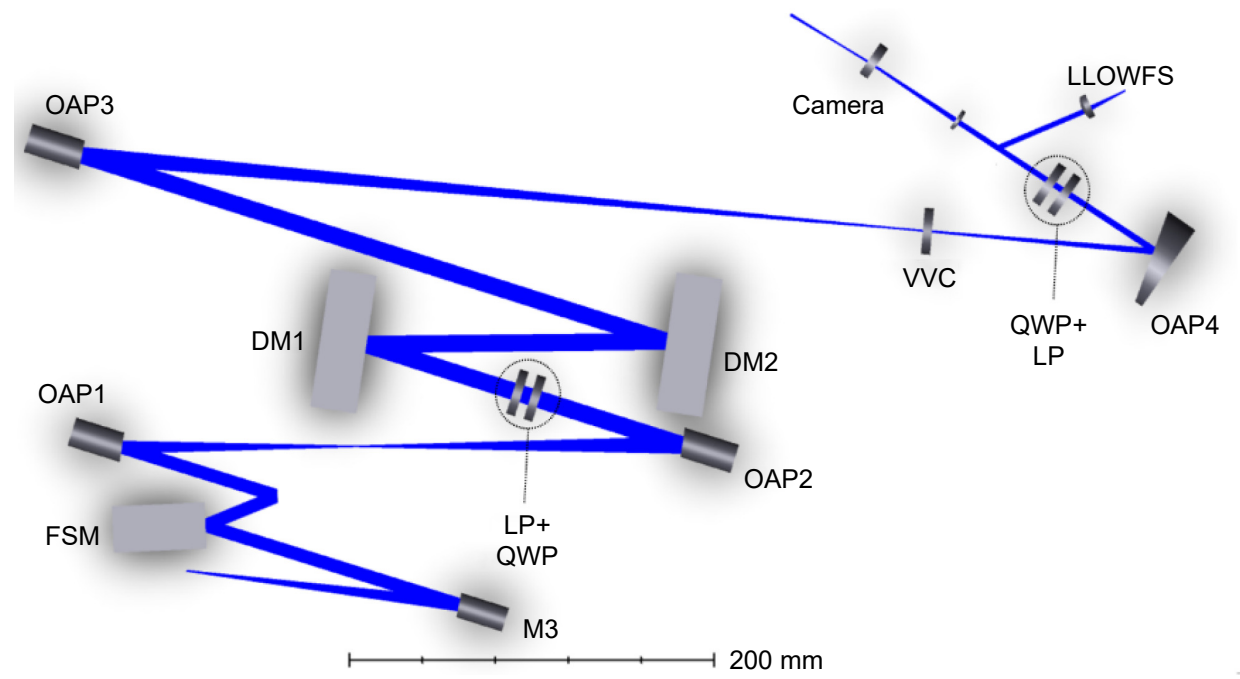

Fig. 29 | An expanded realization of the CDEEP testbed with two deformable mirrors to study a coronagraph's ability to correct diffraction features that result from heavily segmented pupils (e.g., LUVOIR ${ }^{86}$ ) or secondary support spiders (e.g., Nancy Grace Roman Space Telescope $^{87}$ ) using the Active Correction for Aperture Discontinuities technique ${ }^{88}$. 
the direct detection of debris disks and exoplanets ${ }^{27}$.

To prototype this mission and develop a platform for exploring future high-contrast imaging concepts, a vacuum-compatible testbed based on the optical layout of CDEEP (Fig. 29) is under construction at the University of Arizona. This testbed will serve as the primary platform for testing the CDEEP mission concept, as well as investigations into VVC technology and electric field conjugation focal plane wavefront sensing ${ }^{85}$. The highperformance and compact form factor are ideal for prototyping future high-contrast imaging instrumentation intended for spaceborne observatories. Presently, the optical and mechanical design of the testbed are completed, and construction is slated to begin in 2021. The testbed will begin operations in a cleanroom environment, but ultimately be integrated into a vacuum chamber for further testing and refinement in a relevant environment. The development of the CDEEP testbed marks the beginning of The University of Arizona's journey to developing high-performance optical satellite payloads in support of space astrophysics and planetary science investigations.

\section{Concluding remark}

A diverse selection of ground-based and space-based future telescope technologies are actively being conceptualized, designed, prototyped, and demonstrated at the University of Arizona. Optical polishing enhancements discussed in Section Deterministic computer controlled optical surfacing technologies enable efficient production of future optical elements. New engineering technology in Section Very large telescope control system, instrument, and segmentation will upgrade and expand capabilities of existing very large ground-based or space telescopes. Finally, in Section Future space telescope concepts and enabling technologies, a view of the future shows how space-based telescopes that had previously been only a dream, are now realizable through the advances in optical engineering technologies. This suite of optical technologies serves the next generation of astronomical investigations by offering novel yet applicable approaches that the wider design and engineering community can practically use. It is our hope that these contributions in design and instrumentation will not only provide new practical benchmarks for modern astronomy today, but also precipitate the next great insights and questions about our universe.

\section{References}

1. Martin HM, Allen RG, Burge JH, Kim DW, Kingsley JS et al. Fabrication and testing of the first 8.4-m off-axis segment for the Giant Magellan Telescope. Proc SPIE 7739, 77390A (2010).

2. Martin HM, Allen RG, Burge JH, Kim DW, Kingsley JS et al. Production of $8.4 \mathrm{~m}$ segments for the Giant Magellan Telescope. Proc SPIE 8450, 84502D (2012).

3. Martin HM, Burge JH, Davis JM, Kim DW, Kingsley JS et al. Status of mirror segment production for the Giant Magellan Telescope. Proc SPIE 9912, 99120V (2016).

4. Martin HM, Allen R, Gasho V, Jannuzi BT, Kim DW et al. Manufacture of primary mirror segments for the Giant Magellan Telescope. Proc SPIE 10706, 107060V (2018).

5. Kim DW, Kim SW, Burge JH. Non-sequential optimization technique for a computer controlled optical surfacing process using multiple tool influence functions. Opt Express 17, 21850-21866 (2009).

6. Ke XL, Wang TY, Choi H, Pullen W, Huang $L$ et al. Dual-tool multiplexing model of parallel computer controlled optical surfacing. Opt Lett 45, 6426-6429 (2020).

7. Kim DW, Park WH, Kim SW, Burge JH. Edge tool influence function library using the parametric edge model for computer controlled optical surfacing. Proc SPIE 7426, 74260G (2009).

8. Negi VS, Garg H, Shravan Kumar RR, Karar V, Tiwari UK et al. Parametric removal rate survey study and numerical modeling for deterministic optics manufacturing. Opt Express 28, 26733-26749 (2020).

9. Wang TY, Huang L, Kang $\mathrm{H}$, Choi $\mathrm{H}$, Kim DW et al. RIFTA: a robust iterative Fourier transform-based dwell time Algorithm for ultra-precision ion beam figuring of synchrotron mirrors. Sci Rep 10, 8135 (2020).

10. Wang TY, Huang L, Zhu Y, Vescovi M, Khune D et al. Development of a position-velocity-time-modulated two-dimensional ion beam figuring system for synchrotron x-ray mirror fabrication. Appl Opt 59, 3306-3314 (2020).

11. Rodriguez S, Rakich A, Hill J, Kuhn O, Brendel T et al. Implementation of a laser-truss based telescope metrology system at the Large Binocular Telescope. Proc SPIE 11487, 114870E (2020).

12. Rakich A, Choi H, Veillet C, Hill JM, Bec M et al. A laser-truss based optical alignment system on LBT. Proc SPIE 11445, 114450R (2020).

13. Kang H, Thompson D, Conrad A, Vogel C, Lamdan A et al. Modular plug-in extension enabling cross-dispersed spectroscopy for Large Binocular Telescope. Proc SPIE 11116, 1111606 (2019).

14. Feng YT, Ashcraft JN, Breckinridge JB, Harvey JE, Douglas ES et al. Topological pupil segmentation and point spread function analysis for large aperture imaging systems. Proc SPIE 11568, 115680I (2020).

15. Apai D, Milster TD, Kim DW, Bixel A, Schneider $G$ et al. A thousand earths: a very large aperture, ultralight space telescope array for atmospheric biosignature surveys. Astron $J$ 
158, 83 (2019).

16. Apai D, Milster TD, Arenberg J, Kim D, Liang R et al. Nautilus deep space observatory: a giant segmented space telescope array for a galactic biosignature survey. In Deep Space Gateway Concept Science Workshop 3127 (LPI, 2018). Bibcode: 2018LPICo2063.3127A

17. Apai D, Milster TD, Kim DW, Bixel A, Schneider G et al. Nautilus Observatory: a space telescope array based on very large aperture ultralight diffractive optical elements. Proc SPIE 11116, 1111608 (2019).

18. Milster TD, Apai D, Kim DW, Kim YS, Kim GH et al. Progress toward optical design and fabrication of ultralight, large aperture transmissive lenses for space telescopes. In Frontiers in Optics 2020 FM1A.2 (OSA, 2020); https://doi.org/10.1364/ FIO.2020.FM1A.2.

19. Kim DW, Walker CK, Apai D, Milster TD, Takashima $Y$ et al. Disruptive space telescope concepts, designs, and developments: OASIS and Nautilus -INVITED. EPJ Web Conf 238, 06001 (2020).

20. Esparza MA, Choi H, Kim DW. Alignment of Multi-Order Diffractive Engineered (MODE) lens segments using the Kinematically-Engaged Yoke System. Proc SPIE 11487, 114870V (2020).

21. Choi H, Esparza MA, Lamdan A, Feng TT, Milster T et al. Inprocess metrology for segmented optics UV curing control. Proc SPIE 11487, 114870M (2020).

22. Walket CK, Smith IS, Goldsmith PF, O'Dougherty S, Takashima $Y$ et al. Spherical reflectors for space based telescopes. In Proceedings of 2017 IEEE MTT-S International Microwave Symposium (IMS) $1884-1887$ (IEEE, 2017); https://doi.org/ 10.1109/MWSYM.2017.8059024.

23. Chandra A, Sirsi S, Choi H, Phan A, Takashima $Y$ et al. Thermally formed inflatable reflectors for space telescopes. In Proceedings of 2020 IEEE Aerospace Conference 1-9 (IEEE, 2020); https://doi.org/10.1109/AERO47225.2020.9172651.

24. Quach H, Berkson J, Sirsi S, Choi H, Dominguez R et al. Fullaperture optical metrology for inflatable membrane mirrors. Proc SPIE 11487, 114870N (2020).

25. Choi H, Trumper IL, Feng YT, Kang H, Berkson J et al. Longslit cross-dispersion spectroscopy for Hyperion UV space telescope. J Astron Telesc, Instrum, Syst 7, 014006 (2021).

26. Choi H, Trumper I, Feng YT, Kang H, Hamden E et al. Hyperion: far-UV cross dispersion spectroscope design. Proc SPIE 11487, 114870W (2020).

27. Maier ER, Douglas ES, Kim DW, Su K, Ashcraft JN et al. Design of the vacuum high contrast imaging testbed for CDEEP, the Coronagraphic Debris and Exoplanet Exploring Pioneer. Proc SPIE 11443, 114431Y (2020).

28. Kim DW, Esparza M, Quach H, Rodriguez S, Kang H et al. Optical technology for future telescopes. Proc SPIE 11761, 1176103 (2021).

29. Cheng H. Independent Variables for Optical Surfacing Systems: Synthesis, Characterization and Application. (SpringerVerlag, Berlin, 2014).

30. Carnal CL, Egert CM, Hylton KW. Advanced matrix-based al- gorithm for ion-beam milling of optical components. Proc SPIE 1752, 54-62 (1992).

31. Wu JF, Lu ZW, Zhang HX, Wang TS. Dwell time algorithm in ion beam figuring. Appl Opt 48, 3930-3937 (2009).

32. Huang T, Zhao D, Cao ZC. Trajectory planning of optical polishing based on optimized implementation of dwell time. Precis Eng 62, 223-231 (2020).

33. Zhou L, Dai YF, Xie XH, Jiao CJ, Li SY. Model and method to determine dwell time in ion beam figuring. Nanotechnol Precis Eng 5, 107-112 (2007).

34. Zhang YF, Fang FZ, Huang W, Fan W. Dwell time algorithm based on bounded constrained least squares under dynamic performance constraints of machine tool in deterministic optical finishing. Int J Precis Eng Manuf-Green Technol (2021).

35. Jiao CJ, Li SY, Xie XH. Algorithm for ion beam figuring of lowgradient mirrors. Appl Opt 48, 4090-4096 (2009).

36. Wilson SR, McNeil JR. Neutral ion beam figuring of large optical surfaces. Proc SPIE 0818, 320-324 (1987).

37. Wang TY, Huang L, Vescovi M, Kuhne D, Tayabaly $\mathrm{K}$ et al. Study on an effective one-dimensional ion-beam figuring method. Opt Express 27, 15368-15381 (2019).

38. Richardson $\mathrm{WH}$. Bayesian-based iterative method of image restoration. J Opt Soc Am 62, 55-59 (1972).

39. Nelder JA, Mead R. A simplex method for function minimization. Comput J 7, 308-313 (1965).

40. Huang L, Wang TY, Tayabaly K, Kuhne D, Xu WH et al. Stitching interferometry for synchrotron mirror metrology at National Synchrotron Light Source II (NSLS-II). Opt Lasers Eng 124, 105795 (2020).

41. Huang L, Wang TY, Nicolas J, Vivo A, Polack F et al. Two-dimensional stitching interferometry for self-calibration of highorder additive systematic errors. Opt Express 27, 26940-26956 (2019).

42. Heidt J, Thompson D. LUCI Users Manual. September 7, 2016 https://sites.google.com/a/lbto.org/luci/documents-and-links.

43. Werenskiold $\mathrm{CH}$. Improved telescope spider design. J Roy Astron Soc Can 35, 268 (1941).

44. Couder A. Dealing with spider diffraction. In Amateur Telescope Making (Book Two), Ingalls AG, ed, 8th Printing 620-622 (Scientific American, Inc, 1952).

45. Everhart E, Kantorski JW. Diffraction patterns produced by obstructions in reflecting telescopes of modest size. Astron J 64, 455 (1959).

46. Richter JL. Spider diffraction: a comparison of curved and straight legs. Appl Opt 23, 1907-1913 (1984).

47. Harvey JE, Ftaclas C. Diffraction effects of telescope secondary mirror spiders on various image-quality criteria. Appl Opt 34, 6337-6349 (1995).

48. Kasdin NJ, Vanderbei RJ, Spergel DN, Littman MG. Extrasolar planet finding via optimal apodized-pupil and shaped-pupil coronagraphs. Astrophys J 582, 1147-1161 (2003).

49. Breckinridge JB, Harvey JE, Crabtree K, Hull T. Exoplanet telescope diffracted light minimized: the pinwheel-pupil solution. Proc SPIE 10698, 106981P (2018).

50. Snik F, Absil O, Baudoz P, Beaulieu M, Bendek E et al. 
Review of high-contrast imaging systems for current and future ground-based and space-based telescopes III: technology opportunities and pathways. Proc SPIE 10706, 107062L (2018).

51. Breckinridge JB, Harvey JE, Irvin R, Chipman R, Kupinski M et al. ExoPlanet Optics: conceptual design processes for stealth telescopes. Proc SPIE 11115, 111150H (2019).

52. Harvey JE, Breckinridge JB, Irvin RG, Pfisterer RN. Novel designs for minimizing diffraction effects of large segmented mirror telescopes. Proc SPIE 10745, 107450L (2018).

53. Perrin M, Long J, Douglas E, Sivaramakrishnan A, Slocum C et al. POPPY: physical optics propagation in PYthon. Astrophysics Source Code Library, record ascl: 1602.018 (2016).

54. Perrin MD, Soummer R, Elliott EM, Lallo MD, Sivaramakrishnan A. Simulating point spread functions for the James Webb Space Telescope with WebbPSF. Proc SPIE 8442, 84423D (2012).

55. Ruane G, Riggs A, Coker CT, Shaklan SB, Sidick E et al. Fast Linearized Coronagraph Optimizer (FALCO) IV: coronagraph design survey for obstructed and segmented apertures. Proc SPIE 10698, 106984U (2018).

56. N'Diaye M, Soummer R, Pueyo L, Carlotti A, Stark CC et al. Apodized pupil lyot coronagraphs for arbitrary apertures. Astrophys J 818, 163 (2016).

57. Kim Y, Wang ZC, Milster T. Ultralight very large aperture space telescopes using MODE lens technology. In Optical Design and Fabrication 2019 FM4B.4 (OSA, 2019). https://doi.org/10.1364/FREEFORM.2019.FM4B.4

58. Walker C, Kulesa C, Smith IS, Perry B, Takashima Y et al. Orbiting Astronomical Satellite for Investigating Stellar Systems (OASIS): following water from the interstellar medium to oceans. Bulletin of the AAS [Internet]. 2019 Sep 30;51(7). https://baas.aas.org/pub/2020n7i047.

59. Knauer MC, Kaminski J, Hausler G. Phase measuring deflectometry: a new approach to measure specular free-form surfaces. Proc SPIE 5457, 366-376 (2004).

60. Su P, Parks RE, Wang LR, Angel RP, Burge JH. Software configurable optical test system: a computerized reverse Hartmann test. Appl Opt 49, 4404-4412 (2010).

61. Su TQ, Wang SS, Parks RE, Su P, Burge JH. Measuring rough optical surfaces using scanning long-wave optical test system. Appl Opt 52, 7117-7126 (2013).

62. Evans CJ, Kestner RN. Test optics error removal. Appl Opt 35, 1015-1021 (1996).

63. Decataldo D, Pallottini A, Ferrara A, Vallini L, Gallerani S. Photoevaporation of jeans-unstable molecular clumps. Mon Not Roy Astron Soc 487, 3377-3391 (2019).

64. Gould RJ, Salpeter EE. The interstellar abundance of the hydrogen molecule. I. Basic processes. Astrophys J 138, 393 (1963).

65. Krumholz MR. The big problems in star formation: the star formation rate, stellar clustering, and the initial mass function. Phys Rep 539, 49-134 (2014).

66. Smith WJ. Modern Lens Design 2nd ed (McGraw-Hill, New York, 2005).
67. Shannon RR. The Art and Science of Optical Design (Cambridge University Press, Cambridge, 1997).

68. Trumper I, Anderson AQ, Howard JM, West G, Kim DW. Design form classification of two-mirror unobstructed freeform telescopes. Opt Eng 59, 025105 (2020).

69. West SC, Bailey SH, Bauman S, Cuerden B, Granger Z et al. A space imaging concept based on a $4 \mathrm{~m}$ structured spun-cast borosilicate monolithic primary mirror. Proc SPIE 7731, 773110 (2010).

70. Allan G, Douglas ES, Barnes D, Egan M, Furesz G et al. The deformable mirror demonstration mission (DeMi) CubeSat: optomechanical design validation and laboratory calibration. Proc SPIE 10698, 1069857 (2018).

71. Morgan RE, Douglas ES, Allan GW, Bierden P, Chakrabarti S et al. MEMS deformable mirrors for space-based high-contrast imaging. Micromachines 10, 366 (2019).

72. Mendillo CB, Howe GA, Hewawasam K, Martel J, Finn SC et al. Optical tolerances for the PICTURE-C mission: error budget for electric field conjugation, beam walk, surface scatter, and polarization aberration. Proc SPIE 10400, 1040010 (2017).

73. Mendillo CB, Hicks BA, Cook TA, Bifano TG, Content DA et al. PICTURE: a sounding rocket experiment for direct imaging of an extrasolar planetary environment. ProC SPIE 8442, 84420E (2012).

74. Chakrabarti S, Mendillo CB, Cook TA, Martel JF, Finn SC et al. Planet Imaging Coronagraphic Technology Using a Reconfigurable Experimental Base (PICTURE-B): the second in the series of suborbital exoplanet experiments. J Astron Inst 5, 1640004 (2016).

75. Douglas ES, Mendillo CB, Cook TA, Cahoy KL, Chakrabarti S. Wavefront sensing in space: flight demonstration II of the PICTURE sounding rocket payload. J Astron Telesc, Instrum, Syst 4, 019003 (2018).

76. Belikov R, Lozi J, Pluzhnik E, Hix TT, Bendek E et al. EXCEDE technology development III: first vacuum tests. Proc SPIE 9143, 914323 (2014).

77. Sirbu D, Thomas SJ, Belikov R, Lozi J, Bendek E et al. EXCEDE technology development IV: demonstration of polychromatic contrast in vacuum at $1.2 \mathrm{~N} / \mathrm{D}$. Proc SPIE 9605, 96050J (2015).

78. Tinker F, Ragan C, Bodden A, Dahlberg K. 2016. Silicon Carbide Optical Telescopes in "Small Satellite" Constellations. AIAA/USU Conference on Small Satellites, August. https://digitalcommons.usu.edu/smallsat/2016/Poster3/4.

79. Tinker F, Xin K. Fabrication of SiC aspheric mirrors with low mid-spatial error. Proc SPIE 8837, 88370M (2013).

80. Mawet D, Serabyn E, Liewer K, Hanot C, McEldowney S et al. Optical vectorial vortex coronagraphs using liquid crystal polymers: theory, manufacturing and laboratory demonstration. Opt Express 17, 1902-1918 (2009).

81. Mawet D, Serabyn E, Liewer K, Burruss R, Hickey J et al. The vector vortex coronagraph: laboratory results and first light at Palomar observatory. Astrophys J 709, 53-57 (2010).

82. Ruane G, Crill B, Patterson K, Prada CM, Seo BJ et al. Decadal survey testbed commissioning roadmap: demonstrating 
technology for imaging new worlds. Jet Propulsion Laboratory, NASA ExEP Program roadmap, 11 (2019).

83. Serabyn G, Mawet D, Ruane G, Mejia-Prada C, Jovanovic N. Technology development for exoplanet MISSONS: technology milestone white paper vortex coronagraph technology. TDEM White Paper, JPL TDEM Whitepaper (2019).

84. Singh G, Martinache F, Baudoz P, Guyon O, Matsuo T et al. Lyot-based low order wavefront sensor for phase-mask coronagraphs: principle, simulations and laboratory experiments. Publ Astron Soc Pac 126, 586-594 (2014).

85. Belikov R, Give'on A, Kern B, Cady E, Carr M et al. Demonstration of high contrast in $10 \%$ broadband light with the shaped pupil coronagraph. Proc SPIE 6693, 66930Y (2007).

86. Pueyo L, Stark C, Juanola-Parramon R, Zimmerman N, Bolcar $M$ et al. The LUVOIR Extreme Coronagraph for Living Planetary Systems (ECLIPS) I: searching and characterizing exoplanetary gems. Proc SPIE 11117, 1111703 (2019).

87. Kasdin NJ, Bailey VP, Mennesson B, Zellem RT, Ygouf M et al. The Nancy grace roman space telescope coronagraph instrument (CGI) technology demonstration. ProC SPIE 11443, 114431U (2020).

88. Mazoyer J, Pueyo L. Fundamental limits to high-contrast wavefront control. Proc SPIE 10400, 1040014 (2017).

\section{Acknowledgements}

The authors would like to acknowledge the Gordon and Betty Moore Foundation for their financial support of the development of the MODE lens and its enabling alignment technologies. Also, we would like to acknowledge the II-VI Foundation Block-Gift, Technology Research Initiative Fund Optics/Imaging Program, and Friends of Tucson Optics Endowed Scholarships in Optical Sciences for helping support the optical design and metrology research conducted in the LOFT group. We also thank Prof. Greg Smith for his technical and editorial inputs and helps during the manuscript writing process.

\section{Author contributions}

D. Kim supervised/co-supervised the overall optical engineering projects presented in this review summary paper. H. Choi co-supervised overall projects including the LBT prime focus camera alignment using laser truss alignment system and the Nautilus MODE lens segment UV curing system, and designed the long-slit cross-dispersion spectroscopy of Hyperion space telescope. T. Brendel co-developed and summarized the LBT prime focus camera alignment methodology using laser truss alignment system. $\mathrm{H}$. Quach co-developed the OASIS inflatable reflector metrology system for the terahertz very large space observatory. M. Esparza co-developed the Nautilus segmented transmissive optics alignment technology for MODE lens. H. Kang co-designed and assembled the LBT MOBIUS cross-dispersion unit. Y. T. Feng performed the pupil segmentation topology simulations and investigated the point spread functions. J. N. Ashcraft co-designed the CDEEP space coronagraph telescope's optical system and its TVAC testbed system. X. L. Ke and T. Y. Wang researched and co-investigated the CCOS run multiplexing theory and dwell time optimization algorithm. E. S. Douglas is the PI of the CDEEP project and co-edited this review summary manuscript with $\mathrm{D}$. Kim.

\section{Competing interests}

The authors declare no competing financial interests. 